1 Hacettepe Journal of Mathematics and Statistics

$\bigcap$ Volume 47 (4) (2018), 855-876

\title{
New results on type-2 soft sets
}

\author{
Khizar Hayat*, Muhammad Irfan Ali† ${ }^{\dagger \ddagger}$ Bing-Yuan $\mathrm{Cao}^{\S \uparrow}$ and Faruk Karaaslan"
}

\begin{abstract}
In this paper, we introduce some new operations on type- 2 soft sets and discuss related properties. The notions of primary empty type- 2 soft sets, underlying empty type- 2 soft sets and complete type- 2 soft sets are introduced. In particular, we define four new operations (the extension, the restriction, the extension-restriction, the restriction-extension) each on union, intersection and difference. By using these new definitions we prove certain De Morgan's laws in type-2 soft set theory. Finally, an example which shows the validity of De Morgan's laws in real life problems is presented.
\end{abstract}

Keywords: Type-2 soft sets, empty type- 2 soft sets, the extension, the restriction, the extension-restriction, the restriction-extension, union, intersection, difference and De Morgan's laws.

Mathematics Subject Classification (2010): Primary: 03B52, 05C72, 08A72;

Secondary: 06D72, 03C05.

Received: 16.02.2017 Accepted:01.06.2017 Doi : 10.15672/HJMS.2017.484

*School of Mathematics and Information Sciences, Guangzhou University, 510000 Guangzhou, China,

Email : khizar233@gmail.com

${ }^{\dagger}$ Department of Mathematics, Islamabad Model College for Girls, F-6/2, Islamabad, Pakistan, Email : mirfanali13@yahoo.com

$\ddagger_{\text {Corresponding Author. }}$

$\S$ School of Mathematics and Information Sciences, Guangzhou University, 510000 Guangzhou, China,

Email : caobingy@163.com

ฯCorresponding Author.

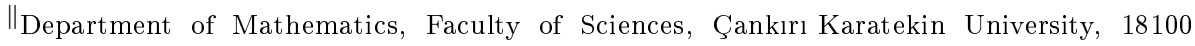
Çankırı, Turkey,

Email : fkaraas lan@karatekin.edu.tr 


\section{Introduction}

Soft set theory [19], firstly initiated by Molodtsov, is a new mathematical tool for dealing with uncertainties. Different from many traditional tools for dealing with uncertainties, such as fuzzy set theory [26], rough set theory [20], intuitionistic fuzzy sets [8] and hesitant fuzzy sets [24], the main advantage of soft set theory is that it is free from the inadequacy of the parametrization tools of those theories. Indeed, Molodtsov [19], shows applicability of soft sets to several fields and provides some fundamental results subsequently augmented by works like Maji et al. [15], Pei and Miao [21] and Aktas and Cagman [1], among others. The soft set theory is a new approach for research of uncertain problems and decision problems. Then, it is applied in many different fields including decision-makings, the smoothness of functions, game theory, operations research, probability theory and theory of measurement. Chen et al. [11], proposed a new definition of soft set parameterization reduction and compared it with attributes reduction in rough set theory.

Ali et al. [2], presented some new operations in soft set theory and based on the analysis of several operations on soft sets Sezgin and Atagün [22], studied the theoretical aspect of operations in soft set theory. In recent decades, the theory of soft sets gone through remarkably rapid strides with wide-ranging applications especially in soft decision making. Maji et al. [17] handled some decision making problems by using soft sets. Cagman and Enginoğlu [9], presented uni-int decision making method and discussed an example which shows the applicability of method in various problems that contain uncertainties. Recently, it has advanced a breadth of the development of information sciences with intelligent systems, expert and decision support systems, self-adaptation and selforganizational systems, information and knowledge, approximate reasoning, modeling and computing with words.

Theory of soft sets and its hybrid models such as rough soft sets and soft rough sets have been successfully applied in algebraic structures [3, 4, 12, 14, 23, 25]. Soft sets have been extended in several directions starting with Maji et al. [16], who initiate fuzzy soft sets. Since then, many authors [5, 7, 18], have studied the fuzzy soft sets in decision makings and fuzzy soft operations as well. With the improvement of soft sets, extended mathematical tools for dealing with incompleteness and uncertainty have been proposed, such as fuzzy parameterized soft set, bipolar soft set, fuzzy soft rough sets and so on, but these tools have the same limitation that there is a lack of freedom in choice of parameters. If there exists a correspondence or an association between parameters, then none of the existing tool can handle the problems entirely. For example, a set of parameters s solid diet, soft diet, fluid diet, calorie rich diet, fibre rich diet, protein rich diet $\}$ describes the characteristic of different food items. It shows that there exist some associations between parameters such that calorie rich diet and protein rich diet consist of some properties in solid diet, soft diet and fluid diet.

In order to deal with associations between parameters, Chatterjee et al. [10], propose the concept of type- 2 soft sets which is a generalization of the Molodtsov's soft sets. It involves parameterization over an already parameterized set and hence has more freedom and efficiency compared to usual soft sets (we termed as type-1 soft sets) in handling impreciseness. Type- 2 soft sets is a new approach to handling uncertainty. Interestingly, Chatterjee et al. [10], investigates some basic operations on type-2 soft sets and uses the model of type-2 soft sets in decision making problems. Also, see [13].

The behavior and the selections of initial parameters and associated parameterized sets corresponding to initial parameters make the study of type- 2 soft sets very interesting. Motivated by this, we consider the operations on type- 2 soft sets and related properties. In this paper, notions of primary empty type- 2 soft sets, underlying empty type- 2 soft 
sets and complete type-2 soft sets are introduced. In particular, we introduced four new operations (the extension, the restriction, the extension-restriction, the restrictionextension) each on union, intersection, and difference. By using these new definitions certain De Morgan's laws are proved in type-2 soft set theory. Finally, an example is presented which shows the validity of De Morgan's laws in real life problems.

\section{Preliminaries}

We adopt the usual description and terminology for soft sets: $U$ denotes a universe of objects, $E$ denotes a universal set of parameters and $A$ is a subset of $E$.

2.1. Definition ([15]). A pair $(F, A)$ is called a soft set over $U$, where $F$ is a mapping given by $F: A \longrightarrow P(U)$.

We refer to Molodtsov's soft sets as type-1 soft sets (briefly $T 1 S S$ ). Note that the set of all $T 1 S S$ over $U$ will be denoted by $\sigma(U)$.

2.2. Definition ([6]). Let $(F, A)$ be a $T 1 S S$ over $U$.

(i) $(F, A)$ is called null $T 1 S S$ if $F(a)=\emptyset$ for all $a \in A$, denoted by $\mathbb{N}_{(U, A)}$.

(ii) A unique $T 1 S S \Phi_{U}$ over $U$ with an empty set of parameters, called the empty $T 1 S S$ over $U$.

(iii) $(F, A)$ is called whole $T 1 S S$ if $F(a)=U$, for all $a \in A$, denoted by $W_{(U, A)}$.

2.3. Definition ([2]). Let $(F, A)$ and $(G, B)$ be two $T 1 S S$ over $U$. Then

(i) The extended union of $(F, A)$ and $(G, B)$, denoted by $(F, A) \widetilde{\cup}_{\varepsilon}(G, B)=(K, C)$, where $C=A \cup B$, is defined $\forall \alpha \in C$, as

$$
K(\alpha)=\left\{\begin{array}{cc}
F(\alpha), & \text { if } \alpha \in A-B \\
G(\alpha), & \text { if } \alpha \in B-A \\
F(\alpha) \cup G(\alpha), & \text { if } \alpha \in A \cap B
\end{array}\right.
$$

(ii) The restricted union of $(F, A)$ and $(G, B)$, denoted by $(F, A) \widetilde{\cup}_{r}(G, B)=(K, C)$, where $C=A \cap B$, is defined $\forall \alpha \in C$, as $F(\alpha) \cup G(\alpha)=K(\alpha)$.

(iii) The extended intersection of $(F, A)$ and $(G, B)$, denoted by $(F, A) \widetilde{\cap}_{\varepsilon}(G, B)=$ $(K, C)$, where $C=A \cup B$, is defined $\forall \alpha \in C$, as

$$
K(\alpha)=\left\{\begin{array}{cc}
F(\alpha), & \text { if } \alpha \in A-B \\
G(\alpha), & \text { if } \alpha \in B-A \\
F(\alpha) \cap G(\alpha), & \text { if } \alpha \in A \cap B
\end{array}\right.
$$

(iv) The restricted intersection of $(F, A)$ and $(G, B)$, denoted by $(F, A) \widetilde{\cap}_{r}(G, B)=$ $(K, C)$, where $C=A \cap B$, is defined $\forall \alpha \in C$, as $F(\alpha) \cap G(\alpha)=K(\alpha)$.

2.4. Definition ([2]). The complement of a $T 1 S S(F, A)$ is denoted by $(F, A)^{2}$ and is defined by $(F, A)^{2}=\left(F^{2}, A\right)$ where $F^{2}: A \longrightarrow P(U)$ is a mapping given by $F^{2}(\alpha)=$ $U-F(\alpha)$ for all $\alpha \in A$.

2.5. Definition. Let $(F, A)$ and $(G, B)$ be two $T 1 S S$ over $U$. The extended difference of $(F, A)$ and $(G, B)$, denoted by $(F, A)-_{\varepsilon}(G, B)=(K, C)$, where $C=A \cup B$, is defined $\forall \alpha \in C$, as

$$
K(\alpha)=\left\{\begin{array}{cc}
F(\alpha), & \text { if } \alpha \in A-B \\
G(\alpha), & \text { if } \alpha \in B-A \\
F(\alpha)-G(\alpha), & \text { if } \alpha \in A \cap B
\end{array}\right.
$$


2.6. Definition ([2]). Let $(F, A)$ and $(G, B)$ be two $T 1 S S$ over $U$. The restricted difference of $(F, A)$ and $(G, B)$, denoted by $(F, A)-_{r}(G, B)=(K, C)$, where $C=A \cap B$, is defined $\forall \alpha \in C$, as $F(\alpha)-G(\alpha)=K(\alpha)$.

2.7. Definition ([10]). Let $(U, E)$ be a soft universe and $\sigma(U)$ be the collection of all $T 1 S S$ over $(U, E)$. Then a mapping $F: A \longrightarrow \sigma(U), A \subseteq E$ is called a type-2 soft set(breifly $T 2 S S)$ over $(U, E)$ and it is denoted by $\left[F^{*}, A\right]$.

In this case, corresponding to each parameter $\alpha \in A, F^{*}(\alpha)$ is a T1SS. Thus, for each $\alpha \in A$, there exists a T1SS, $\left(F_{\alpha}, L_{\alpha}\right)$ such that $F^{*}(\alpha)=\left(F_{\alpha}, L_{\alpha}\right)$ where $F_{\alpha}: L_{\alpha} \longrightarrow P(U)$ and $L_{\alpha} \subset E$. In this case, we refer to the parameter set $A$ as the "primary set of parameters", while the set of parameters $\cup L_{\alpha}$ is known as the "underlying set of parameters".

In order to make this idea clear an example is given in the following.

2.8. Example. Let the universe $U$ under consideration be the set of some food items where

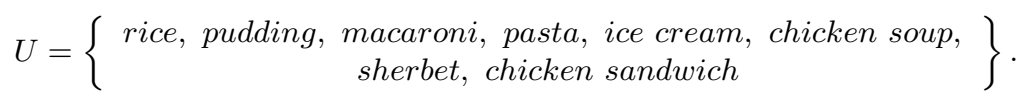

Let $E$ be the set of parameters defined as,

$$
E=\left\{\begin{array}{c}
\text { solid diet, soft diet, fluid diet, calorie rich diet, fibre } \\
\text { rich diet, protein rich diet, carbohydrate rich diet }
\end{array}\right\} .
$$

Suppose,

$$
A=\{\text { calorie rich diet, fibre rich diet, carbohydrate rich diet }\} .
$$

Then, $A \subset E$.

Let $\left[F^{*}, A\right]$ be a $T 2 S S$ over $U$, which denotes the characteristics of the above mentioned food items in terms of food value. We defined as

$$
\begin{aligned}
& F^{*}(\text { calorie rich diet })=\left\{\frac{\text { solid diet }}{\{\text { macaroni, rice, pasta }\}}, \frac{\text { soft diet }}{\{\text { pudding, chicken soup }\}}\right\} \\
& F^{*}(\text { fibre rich diet })=\left\{\frac{\text { solid diet }}{\{\text { chicken sandwich, pasta }\}}, \frac{\text { sof } \text { diet }}{\{\text { ice cream, sherbet }\}}\right\} \\
& F^{*}(\text { carbohydrate rich diet })=\left\{\frac{\text { solid diet }}{\{\text { rice. macaroni }\}}\right\} .
\end{aligned}
$$

2.9. Definition ([10]). Let $\left[F^{*}, A\right]$ and $\left[G^{*}, B\right]$ be two $T 2 S S$ over $U$. The union of $\left[F^{*}, A\right]$ and $\left[G^{*}, B\right]$, denoted by $\left[F^{*}, A\right] \sqcup\left[G^{*}, B\right]=\left[H^{*}, C\right]$, where $C=A \cup B$, is defined $\forall \alpha \in C$, as

$$
H^{*}(\alpha)=\left\{\begin{array}{cc}
F^{*}(\alpha), & \text { if } \alpha \in A-B \\
G^{*}(\alpha), & \text { if } \alpha \in B-A \\
F^{*}(\alpha) \widetilde{\cup} G^{*}(\alpha), & \text { if } \alpha \in A \cap B
\end{array}\right.
$$

where $F^{*}(\alpha) \widetilde{\cup} G^{*}(\alpha)$ for all $\alpha \in A \cap B$, refers to the usual type-1 soft union between the respective $T 1 S S$ corresponding to $F^{*}(\alpha)$ and $G^{*}(\alpha)$ respectively.

2.10. Definition ([10]). Let $\left[F^{*}, A\right]$ and $\left[G^{*}, B\right]$ be two $T 2 S S$ over $U$. The intersection of $\left[F^{*}, A\right]$ and $\left[G^{*}, B\right]$, denoted by $\left[F^{*}, A\right] \sqcap\left[G^{*}, B\right]=\left[H^{*}, C\right]$, where $C=A \cap B$, is defined $\forall \alpha \in C$, as $H^{*}(\alpha)=F^{*}(\alpha) \widetilde{\cap} G^{*}(\alpha)$, where $F^{*}(\alpha) \widetilde{\cap} G^{*}(\alpha)$ for all $\alpha \in A \cap B$, refers to the usual type- 1 soft intersection between the respective $T 1 S S$ corresponding to $F^{*}(\alpha)$ and $G^{*}(\alpha)$ respectively. 
2.11. Definition[10]. Let $\left[F^{*}, A\right]$ and $\left[G^{*}, B\right]$ be two $T 2 S S$ over common universe $U$. Then $\left[F^{*}, A\right]$ is called a type-2 soft subset of $\left[G^{*}, B\right]$ if and only if $A \subset B$, and $F^{*}(\alpha) \subset$ $G^{*}(\alpha) \forall \alpha \in A$, where $F^{*}(\alpha) \subset G^{*}(\alpha)$, refers to the containment of $T 1 S S$ corresponding to $F^{*}(\alpha)$ in $T 1 S S$ corresponding to $G^{*}(\alpha)$. It is denoted as $\left[F^{*}, A\right] \sqsubset\left[G^{*}, B\right]$.

2.12. Definition[10]. The complement of a $T 2 S S\left[F^{*}, A\right]$ is denoted as $\left[F^{*}, A\right]^{2}$ and is defined by $\left[F^{*}, A\right]^{2}=\left[\left(F^{*}\right)^{2}, A\right]$ where $\left(F^{*}\right)^{2}(\alpha)=\left(F_{\alpha}, L_{\alpha}\right)^{2}, \forall \alpha \in A$ such that $F_{\alpha}^{2}(\beta)=$ $U-F_{\alpha}(\beta) \forall \beta \in L_{\alpha}$.

\section{Some new operations on type-2 soft sets}

In Definition 2.2, defined that the empty $T 1 S S$ and the null $T 1 S S$ are two different concepts. In $T 2 S S$ theory, the empty $T 2 S S$ and the null $T 2 S S$ are also two different concepts, but there are two empty $T 2 S S$ appearing one with respect to an empty set of primary parameters and other with respect to empty set of the underlying parameters. We differentiate these two empty $T 2 S S$ as follows:

3.1. Definition. Let $U$ be an initial universe set, $E$ be the universe set of parameters and $A \subset E$.

(i) $\left[F^{*}, A\right]$ is called an null $T 2 S S$ if and only if for each parameter $\alpha \in A$, the T1SS corresponding to $F^{*}(\alpha)$ is a null $T 1 S S$. A null $T 2 S S$ is denoted by $\mathbb{N}_{A}^{*}$.

(ii) We shall denote $\emptyset_{U}^{*}$ by the unique $T 2 S S$ over $(U, E)$ with an empty set of primary parameters which is called the primary empty $T 2 S S$ over $U$.

(iii) $\left[F^{*}, A\right]$ is called an underlying empty $T 2 S S$ if and only if for each $\alpha \in A$, the $T 1 S S$ corresponding to $F^{*}(\alpha)$ is an empty $T 1 S S$. An underlying empty $T 2 S S$ is denoted by $\varnothing_{A}^{*}$.

(iv) $\left[F^{*}, A\right]$ over $U$ is called an absolute $T 2 S S$ if and only if for each $\alpha \in A$, the $T 1 S S$ corresponding to $F^{*}(\alpha)$ is an absolute $T 1 S S$. An absolute $T 2 S S$ is denoted by $W_{A}^{*}$.

3.2. Remarks. By the definition of $T 2 S S, F^{*}(\alpha)=\left(F_{\alpha}, L_{\alpha}\right)$ where $L_{\alpha} \subset E$ for each $\alpha \in A$ and $\Omega=\cup L_{\alpha}$. Then

(i) We may say that $\Omega$ a universal set of underlying parameters and $L_{\alpha}$ is an underlying set of parameters under $T 1 S S$ corresponding to $F^{*}(\alpha)$ for each $\alpha \in A$.

(ii) We denote $F^{*}(\alpha)=\left(F_{\alpha}, L_{\alpha}\right), \forall \alpha \in A$ as $F^{*}(\alpha)=\left\{\frac{\gamma}{F_{\alpha}(\gamma)} \mid \gamma \in L_{\alpha}\right\}$.

3.3. Definition. A $T 2 S S\left[F^{*}, A\right]$ over $U$ is called parallel if and only if for each $\alpha \in A$, the underlying set of parameters of $T 1 S S$ corresponding to $F^{*}(\alpha)$ is equal to $\Lambda$, i.e $\Lambda \subset \Omega$. A parallel $T 2 S S$ is denoted by $\chi_{A}^{*}$.

3.4. Definition. Let $\left[F^{*}, A\right]$ be a $T 2 S S$ over $U$ and $\Omega$ be a set of all underlying parameters. Then $\left[F^{*}, A\right]$ is called an underlying absolute $T 2 S S$ if for each $\alpha \in A$, the underlying set of parameters of $T 1 S S$ corresponding to $F^{*}(\alpha)$ is equal to $\Omega$. An underlying absolute $T 2 S S$ of $A$ is denoted by $\pi_{A}^{*}$.

3.5. Lemma. If $\left[F^{*}, A\right]$ is a underlying absolute $T 2 S S$ of $A$, then $\left[F^{*}, A\right]$ is a parallel $T 2 S S$. 
3.6. Definition. Let $\left[F^{*}, A\right]=\pi_{A}^{*}$ be an underlying absolute $T 2 S S$ over $U$. Then $\pi_{A}^{*}$ is called a complete $T 2 S S$ if and only if for each $\alpha \in A$, the T1SS corresponding to $F^{*}(\alpha)$ is an absolute $T 1 S S$. A complete $T 2 S S$ of $A$ is denoted by $\omega_{A}^{*}=\left[U^{*}, A\right]$.

In Definition 2.9 and Definition 2.10, union and intersection of $T 2 S S$ are defined respectively. As seen in [10]. We may write the Definition 2.9 and the Definition 2.10, as in the following form,

3.7. Definition. Let $\left[F^{*}, A\right]$ and $\left[G^{*}, B\right]$ be two $T 2 S S$ over a common universe $U$. The extended union of $\left[F^{*}, A\right]$ and $\left[G^{*}, B\right]$, denoted by $\left[F^{*}, A\right] \sqcup_{\varepsilon}\left[G^{*}, B\right]=\left[I^{*}, C\right]$, where $C=A \cup B$, is defined $\forall \alpha \in C$, as

$$
I^{*}(\alpha)=\left\{\begin{array}{cc}
F^{*}(\alpha), & \text { if } \alpha \in A-B \\
G^{*}(\alpha), & \text { if } \alpha \in B-A \\
F^{*}(\alpha) \widetilde{\cup}_{\varepsilon} G^{*}(\alpha), & \text { if } \alpha \in A \cap B
\end{array}\right.
$$

It may be mentioned here that

$$
F^{*}(\alpha) \widetilde{\cup}_{\varepsilon} G^{*}(\alpha)=\left\{\begin{array}{cl}
\frac{\gamma}{F_{\alpha}(\gamma)}, & \text { if } \gamma \in L_{\alpha} \\
\frac{\gamma}{G_{\alpha}(\gamma)}, & \text { if } \gamma \in M_{\alpha} \\
\frac{\gamma}{F_{\alpha}(\gamma) \cup G_{\alpha}(\gamma)}, & \text { if } \gamma \in L_{\alpha} \cap M_{\alpha}
\end{array}\right.
$$

for all $\alpha \in C$, where $L_{\alpha}$ and $M_{\alpha}$ are underlying set of parameters of $T 1 S S\left(F_{\alpha}, L_{\alpha}\right)$ and $\left(G_{\alpha}, M_{\alpha}\right)$ corresponding to $F^{*}(\alpha)$ and $G^{*}(\alpha)$ respectively.

In other words $F^{*}(\alpha) \widetilde{\cup}_{\varepsilon} G^{*}(\alpha)$ for all $\alpha \in C$, refers to the extended type-1 soft union between the respective $T 1 S S$ corresponding to $F^{*}(\alpha)$ and $G^{*}(\alpha)$ respectively.

3.8. Definition. Let $\left[F^{*}, A\right]$ and $\left[G^{*}, B\right]$ be two $T 2 S S$ over a common universe $U$. The restricted intersection of $\left[F^{*}, A\right]$ and $\left[G^{*}, B\right]$, denoted by $\left[F^{*}, A\right] \cap_{r}\left[G^{*}, B\right]=\left[I^{*}, C\right]$, where $C=A \cap B \neq \emptyset$, is defined $\forall \alpha \in C$, as $F^{*}(\alpha) \widetilde{\cap}_{r} G^{*}(\alpha)=I^{*}(\alpha)$. It may be mentioned here that

$$
F^{*}(\alpha) \widetilde{\cap}_{r} G^{*}(\alpha)=\left\{\frac{\gamma}{F_{\alpha}(\gamma) \cap G_{\alpha}(\gamma)} \quad \text { if } \gamma \in L_{\alpha} \cap M_{\alpha},\right.
$$

where $L_{\alpha}$ and $M_{\alpha}$ are underlying set of parameters of $\operatorname{T1SS}\left(F_{\alpha}, L_{\alpha}\right)$ and $\left(G_{\alpha}, M_{\alpha}\right)$ corresponding to $F^{*}(\alpha)$ and $G^{*}(\alpha)$ respectively.

In other words $F^{*}(\alpha) \widetilde{\cap}_{r} G^{*}(\alpha)$ for all $\alpha \in C$, refers to the restricted type-1 soft intersection between the respective $T 1 S S$ corresponding to $F^{*}(\alpha)$ and $G^{*}(\alpha)$ respectively.

In addition, we define following definitions of union and intersection.

3.9. Definition. Let $\left[F^{*}, A\right]$ and $\left[G^{*}, B\right]$ be two $T 2 S S$ over a common universe $U$. The restricted union of $\left[F^{*}, A\right]$ and $\left[G^{*}, B\right]$, denoted by $\left[F^{*}, A\right] \sqcup_{r}\left[G^{*}, B\right]=\left[K^{*}, C\right]$, where $C=A \cap B \neq \emptyset$, is defined $\forall \alpha \in C$, as $F^{*}(\alpha) \widetilde{\cup}_{r} G^{*}(\alpha)=K^{*}(\alpha)$, where $F^{*}(\alpha) \widetilde{\cup}_{r} G^{*}(\alpha)$ for all $\alpha \in A \cap B$, refers to the restricted type-1 soft union between the respective $T 1 S S$ corresponding to $F^{*}(\alpha)$ and $G^{*}(\alpha)$ respectively. 
3.10. Definition. Let $\left[F^{*}, A\right]$ and $\left[G^{*}, B\right]$ be two $T 2 S S$ over over a common universe $U$. The extended intersection of $\left[F^{*}, A\right]$ and $\left[G^{*}, B\right]$, denoted by $\left[F^{*}, A\right] \cap_{\varepsilon}\left[G^{*}, B\right]=$ $\left[H^{*}, C\right]$, where $C=A \cup B$, is defined $\forall \alpha \in C$ as

$$
H^{*}(\alpha)=\left\{\begin{array}{cc}
F^{*}(\alpha), & \text { if } \alpha \in A-B \\
G^{*}(\alpha), & \text { if } \alpha \in B-A \\
F^{*}(\alpha) \widetilde{\cap}_{\varepsilon} G^{*}(\alpha), & \text { if } \alpha \in A \cap B
\end{array}\right.
$$

where $F^{*}(\alpha) \widetilde{\cap}_{\varepsilon} G^{*}(\alpha)$ for all $\alpha \in A \cap B$, refers to the extended type-1 soft intersection between the respective $T 1 S S$ corresponding to $F^{*}(\alpha)$ and $G^{*}(\alpha)$ respectively.

3.11. Lemma. Let $\left[F^{*}, A\right]$ and $\left[G^{*}, B\right]$ be two $T 2 S S$ over over a common universe $U$. Then extended intersection, restricted intersection, extended union and restricted union of $\left[F^{*}, A\right]$ and $\left[G^{*}, B\right]$ is also a $T 2 S S$.

Proof. Straightforward.

Let us illustrate above definitions in the following example

3.12. Example. Let $U=\left\{h_{1}, h_{2}, h_{3}, h_{4}, h_{5}, h_{6}, h_{7}\right\}$ be a set of houses under considerations,

$$
E=\left\{\begin{array}{c}
\text { in good location, expensive, beautiful, cheep, wooden, best school } \\
\text { district, with good security, near to market, with pool, furnished }
\end{array}\right\}
$$

be a set of parameters.

Let, $A=\{$ in good location, cheep, best school district $\}$

and $B=\{$ furnished, cheep, best school district $\}$. Then $A, B \subset E$. Let $\left[F^{*}, A\right]$ and $\left[G^{*}, B\right]$ be two $T 2 S S$ over $(U, E)$, defined by

$F^{*}($ in good location $)=\left\{\frac{\text { near to market }}{\left\{h_{2}, h_{3}, h_{7}\right\}}, \frac{\text { beautiful }}{\left\{h_{1}, h_{3}, h_{4}, h_{6}\right\}}\right\}$

$F^{*}($ cheep $)=\left\{\frac{\text { wooden }}{\left\{h_{2}, h_{3}, h_{7}\right\}}, \frac{\text { beautiful }}{\left\{h_{1}, h_{5}, h_{7}\right\}}, \frac{\text { with good security }}{\left\{h_{1}, h_{3}\right\}}\right\}$

$F^{*}($ best school district $)=\left\{\frac{\text { near to market }}{\left\{h_{2}, h_{3}, h_{7}\right\}}, \frac{\text { wooden }}{\left\{h_{2}, h_{4}, h_{5}\right\}}, \frac{\text { beautiful }}{\left\{h_{1}, h_{3}, h_{4}, h_{6}\right\}}\right\}$

$G^{*}($ furnished $)=\left\{\frac{\text { wooden }}{\left\{h_{1}, h_{2}, h_{7}\right\}}, \frac{\text { beautiful }}{\left\{h_{5}, h_{6}\right\}}\right\}$

$G^{*}($ cheep $)=\left\{\frac{\text { wooden }}{\left\{h_{2}, h_{5}, h_{7}\right\}}, \frac{\text { beautiful }}{\left\{h_{1}, h_{3}, h_{5}, h_{6}\right\}}, \frac{\text { near to market }}{\left\{h_{2}, h_{6}\right\}}\right\}$

$G^{*}$ (best school district) $=\left\{\frac{\text { near to market }}{\left\{h_{3}, h_{4}, h_{7}\right\}}, \frac{\text { with pool }}{\left\{h_{2}, h_{5}, h_{6}\right\}}, \frac{\text { beautiful }}{\left\{h_{1}, h_{2}, h_{6}\right\}}\right\}$

Then,

Extended Union $\left[F^{*}, A\right] \sqcup_{\varepsilon}\left[G^{*}, B\right]=\left[I^{*}, C\right]$, where $C=A \cup B$. For all $\alpha \in C$, $\left[I^{*}, C\right]$ is as the following,

$I^{*}($ in good location $)=\left\{\frac{\text { near to market }}{\left\{h_{2}, h_{3}, h_{7}\right\}}, \frac{\text { beautiful }}{\left\{h_{1}, h_{3}, h_{4}, h_{6}\right\}}\right\}$

$I^{*}($ cheep $)=\left\{\begin{array}{c}\frac{\text { wooden }}{\left\{h_{2}, h_{3}, h_{5}, h_{7}\right\}}, \frac{\text { beautiful }}{\left\{h_{1}, h_{3}, h_{5}, h_{6}, h_{7}\right\}}, \frac{\text { with good security }}{\left\{h_{1}, h_{3}\right\}}, \\ \frac{\text { near to market }}{\left\{h_{2}, h_{6}\right\}}\end{array}\right\}$ 
$I^{*}($ best school district $)=\left\{\begin{array}{l}\frac{\text { near to market }}{\left\{h_{2}, h_{3}, h_{4}, h_{7}\right\}}, \frac{\text { beautiful }}{\left\{h_{1}, h_{2}, h_{3}, h_{4}, h_{6}\right\}}, \frac{\text { with pool }}{\left\{h_{2}, h_{5}, h_{6}\right\}}, \\ \frac{\text { wooden }}{\left\{h_{2}, h_{4}, h_{5}\right\}}\end{array}\right\}$

$I^{*}($ furnished $)=\left\{\frac{\text { wooden }}{\left\{h_{1}, h_{2}, h_{7}\right\}}, \frac{\text { beautiful }}{\left\{h_{5}, h_{6}\right\}}\right\}$.

Restricted Intersection $\left[F^{*}, A\right] \sqcap_{r}\left[G^{*}, B\right]=\left[J^{*}, C\right]$, where $C=A \cap B$. For all $\alpha \in C,\left[J^{*}, C\right]$ is as the following,

$J^{*}($ cheep $)=\left\{\frac{\text { wooden }}{\left\{h_{2}, h_{7}\right\}}, \frac{\text { beautiful }}{\left\{h_{1}, h_{5}\right\}}\right\}$

$J^{*}$ (best school district $)=\left\{\frac{\text { near to market }}{\left\{h_{3}, h_{7}\right\}}, \frac{\text { beautiful }}{\left\{h_{1}, h_{6}\right\}}\right\}$.

Restricted Union $\left[F^{*}, A\right] \sqcup_{r}\left[G^{*}, B\right]=\left[K^{*}, C\right]$, where $C=A \cap B$. For all $\alpha \in C$, $\left[K^{*}, C\right]$ is as the following,

$K^{*}($ cheep $)=\left\{\frac{\text { wooden }}{\left\{h_{2}, h_{3}, h_{5}, h_{7}\right\}}, \frac{\text { beautiful }}{\left\{h_{1}, h_{3}, h_{5}, h_{6}, h_{7}\right\}}\right\}$

$K^{*}$ (best school district) $=\left\{\frac{\text { near to market }}{\left\{h_{2}, h_{3}, h_{4}, h_{7}\right\}}, \frac{\text { beautiful }}{\left\{h_{1}, h_{2}, h_{3}, h_{4}, h_{6}\right\}}\right\}$.

Extended Intersection $\left[F^{*}, A\right] \cap_{\varepsilon}\left[G^{*}, B\right]=\left[H^{*}, C\right]$, where $C=A \cup B$. For all $\alpha \in C,\left[H^{*}, C\right]$ is as the following,

$H^{*}($ in good location $)=\left\{\frac{\text { near to market }}{\left\{h_{2}, h_{3}, h_{7}\right\}}, \frac{\text { beautiful }}{\left\{h_{1}, h_{3}, h_{4}, h_{6}\right\}}\right\}$

$H^{*}($ cheep $)=\left\{\frac{\text { wooden }}{\left\{h_{2}, h_{7}\right\}}, \frac{\text { beautiful }}{\left\{h_{1}, h_{5}\right\}}, \frac{\text { with good security }}{\left\{h_{1}, h_{3}\right\}}, \frac{\text { near to market }}{\left\{h_{2}, h_{6}\right\}}\right\}$

$H^{*}$ (best school district) $=\left\{\frac{\text { near to market }}{\left\{h_{3}, h_{7}\right\}}, \frac{\text { with pool }}{\left\{h_{2}, h_{5}, h_{6}\right\}}, \frac{\text { wooden }}{\left\{h_{2}, h_{4}, h_{5}\right\}}, \frac{\text { beautiful }}{\left\{h_{1}, h_{6}\right\}}\right\}$

$H^{*}($ furnished $)=\left\{\frac{\text { wooden }}{\left\{h_{1}, h_{2}, h_{7}\right\}}, \frac{\text { beautiful }}{\left\{h_{5}, h_{6}\right\}}\right\}$.

In the above definitions, a restriction is contemplated over a restriction and an extension is contemplated over an extension. We dealt with the primary parameters and corresponding sets of underlying parameters in the same manner. Interestingly, sometimes primary parameters behaves differently from the corresponding sets of underlying parameters in a type- 2 soft set. In this regard, we now investigate some additional situations such that restriction-extension and extension-restriction. We introduce the following new notions of union and intersection.

3.13. Definition. Let $\left[F^{*}, A\right]$ and $\left[G^{*}, B\right]$ be two $T 2 S S$ over a common universe $U$. The extended-restricted union of $\left[F^{*}, A\right]$ and $\left[G^{*}, B\right]$, denoted by $\left[F^{*}, A\right] \sqcup_{\varepsilon-r}\left[G^{*}, B\right]=$ $\left[I^{*}, C\right]$, where $C=A \cup B$, is defined $\forall \alpha \in C$, as

$$
I^{*}(\alpha)=\left\{\begin{array}{cc}
F^{*}(\alpha), & \text { if } \alpha \in A-B \\
G^{*}(\alpha), & \text { if } \alpha \in B-A \\
F^{*}(\alpha) \widetilde{\cup}_{r} G^{*}(\alpha), \quad \text { if } \alpha \in A \cap B
\end{array}\right.
$$

where $F^{*}(\alpha) \widetilde{\cup}_{r} G^{*}(\alpha)$ for all $\alpha \in A \cap B$, refers to the restricted type-1 soft union between the respective $T 1 S S$ corresponding to $F^{*}(\alpha)$ and $G^{*}(\alpha)$ respectively. 
3.14. Definition. Let $\left[F^{*}, A\right]$ and $\left[G^{*}, B\right]$ be two $T 2 S S$ over a universe $U$. The extendedrestricted intersection of $\left[F^{*}, A\right]$ and $\left[G^{*}, B\right]$, denoted by $\left[F^{*}, A\right] \sqcap_{\varepsilon-r}\left[G^{*}, B\right]=$ $\left[H^{*}, C\right]$, where $C=A \cup B$, is defined $\forall \alpha \in C$ as

$$
H^{*}(\alpha)=\left\{\begin{array}{cc}
F^{*}(\alpha), & \text { if } \alpha \in A-B \\
G^{*}(\alpha), & \text { if } \alpha \in B-A \\
F^{*}(\alpha) \widetilde{\cap}_{r} G^{*}(\alpha), & \text { if } \alpha \in A \cap B
\end{array}\right.
$$

where $F^{*}(\alpha) \widetilde{\cap}_{r} G^{*}(\alpha)$ for all $\alpha \in A \cap B$, refers to the restricted type-1 soft intersection between the respective $T 1 S S$ corresponding to $F^{*}(\alpha)$ and $G^{*}(\alpha)$ respectively.

3.15. Definition. Let $\left[F^{*}, A\right]$ and $\left[G^{*}, B\right]$ be two $T 2 S S$ over a universe $U$. The restrictedextended union of $\left[F^{*}, A\right]$ and $\left[G^{*}, B\right]$, denoted by $\left[F^{*}, A\right] \sqcup_{r-\varepsilon}\left[G^{*}, B\right]=\left[J^{*}, C\right]$, where $C=A \cap B \neq \emptyset$, is defined $\forall \alpha \in C$, as $F^{*}(\alpha) \widetilde{\cup}_{\varepsilon} G^{*}(\alpha)=J^{*}(\alpha)$, where $F^{*}(\alpha) \widetilde{\cup}_{\varepsilon} G^{*}(\alpha)$ for all $\alpha \in A \cap B$, refers to the extended type-1 soft union between the respective $T 1 S S$ corresponding to $F^{*}(\alpha)$ and $G^{*}(\alpha)$ respectively.

3.16. Definition. Let $\left[F^{*}, A\right]$ and $\left[G^{*}, B\right]$ be two $T 2 S S$ over a universe $U$. The restrictedextended intersection of $\left[F^{*}, A\right]$ and $\left[G^{*}, B\right]$, denoted by $\left[F^{*}, A\right] \cap_{r-\varepsilon}\left[G^{*}, B\right]=\left[K^{*}, C\right]$, where $C=A \cap B$, is defined $\forall \alpha \in C$, as $F^{*}(\alpha) \widetilde{\cap}_{\epsilon} G^{*}(\alpha)=K^{*}(\alpha)$, where $F^{*}(\alpha) \widetilde{\cap}_{\varepsilon} G^{*}(\alpha)$ for all $\alpha \in A \cap B$, refers to the extended type-1 soft intersection between the respective $T 1 S S$ corresponding to $F^{*}(\alpha)$ and $G^{*}(\alpha)$ respectively.

3.17. Lemma. Let $\left[F^{*}, A\right]$ and $\left[G^{*}, B\right]$ be two $T 2 S S$ over over a common universe $U$. Then the restricted-extended intersection, extended-restricted intersection, restricted-extended union and extended-restricted union of $\left[F^{*}, A\right]$ and $\left[G^{*}, B\right]$ is also a $T 2 S S$.

Proof. Straightforward.

Let us illustrate above definitions in the following example

3.18. Example. Consider two T2SS $\left[F^{*}, A\right]$ and $\left[G^{*}, B\right]$ defined in the Example 3.12. Then,

Extended-Restricted Union $\left[F^{*}, A\right] \sqcup_{\varepsilon-r}\left[G^{*}, B\right]=\left[I^{*}, C\right]$, where $C=A \cup B$. For all $\alpha \in C,\left[I^{*}, C\right]$ is as the following,

$I^{*}($ in good location $)=\left\{\frac{\text { near to market }}{\left\{h_{2}, h_{3}, h_{7}\right\}}, \frac{\text { beautiful }}{\left\{h_{1}, h_{3}, h_{4}, h_{6}\right\}}\right\}$

$I^{*}($ cheep $)=\left\{\frac{\text { wooden }}{\left\{h_{2}, h_{3}, h_{5}, h_{7}\right\}}, \frac{\text { beautiful }}{\left\{h_{1}, h_{3}, h_{5}, h_{6}, h_{7}\right\}}\right\}$

$I^{*}$ (best school district $)=\left\{\frac{\text { near to market }}{\left\{h_{2}, h_{3}, h_{4}, h_{7}\right\}}, \frac{\text { beautiful }}{\left\{h_{1}, h_{2}, h_{3}, h_{4}, h_{6}\right\}}\right\}$

$I^{*}($ furnished $)=\left\{\frac{\text { wooden }}{\left\{h_{1}, h_{2}, h_{7}\right\}}, \frac{\text { beautiful }}{\left\{h_{5}, h_{6}\right\}}\right\}$.

Extended-Restricted Intersection $\left[F^{*}, A\right] \sqcap_{\varepsilon-r}\left[G^{*}, B\right]=\left[H^{*}, C\right]$, where $C=A \cup$ $B$. For all $\alpha \in C,\left[H^{*}, C\right]$ is as the following,

$H^{*}($ in good location $)=\left\{\frac{\text { near to market }}{\left\{h_{2}, h_{3}, h_{7}\right\}}, \frac{\text { beautiful }}{\left\{h_{1}, h_{3}, h_{4}, h_{6}\right\}}\right\}$

$H^{*}($ cheep $)=\left\{\frac{\text { wooden }}{\left\{h_{2}, h_{7}\right\}}, \frac{\text { beautiful }}{\left\{h_{1}, h_{5}\right\}}\right\}$ 
$H^{*}$ (best school district $)=\left\{\frac{\text { near to market }}{\left\{h_{3}, h_{7}\right\}}, \frac{\text { beautiful }}{\left\{h_{1}, h_{6}\right\}}\right\}$

$H^{*}($ furnished $)=\left\{\frac{\text { wooden }}{\left\{h_{1}, h_{2}, h_{7}\right\}}, \frac{\text { beautiful }}{\left\{h_{5}, h_{6}\right\}}\right\}$.

Restricted-Extended Union $\left[F^{*}, A\right] \sqcup_{r-\varepsilon}\left[G^{*}, B\right]=\left[K^{*}, C\right]$, where $C=A \cap B$. For all $\alpha \in C,\left[K^{*}, C\right]$ is as the following,

$K^{*}($ cheep $)=\left\{\begin{array}{c}\text { wooden } \\ \left\{h_{2}, h_{3}, h_{5}, h_{7}\right\} \\ \frac{\text { beautiful }}{\left\{h_{1}, h_{3}, h_{5}, h_{6}, h_{7}\right\}}, \frac{\text { with good security }}{\left\{h_{1}, h_{3}\right\}}, \\ \frac{\text { near to market }}{\left\{h_{2}, h_{6}\right\}}\end{array}\right\}$
$K^{*}($ best school district $)=\left\{\begin{array}{c}\frac{\text { near to market }}{\left\{h_{2}, h_{3}, h_{4}, h_{7}\right\}}, \frac{\text { with pool }}{\left\{h_{2}, h_{5}, h_{6}\right\}}, \frac{\text { wooden }}{\left\{h_{2}, h_{4}, h_{5}\right\}}, \\ \frac{\text { beautiful }}{\left\{h_{1}, h_{2}, h_{3}, h_{4}, h_{6}\right\}}\end{array}\right\}$

Restricted-Extended Intersection $\left[F^{*}, A\right] \sqcap_{r-\varepsilon}\left[G^{*}, B\right]=\left[J^{*}, C\right]$, where $C=$ $A \cap B$. For all $\alpha \in C,\left[J^{*}, C\right]$ is as the following,

$J^{*}($ cheep $)=\left\{\frac{\text { wooden }}{\left\{h_{2}, h_{7}\right\}}, \frac{\text { beautiful }}{\left\{h_{1}, h_{5}\right\}}, \frac{\text { with good security }}{\left\{h_{1}, h_{3}\right\}}, \frac{\text { near to market }}{\left\{h_{2}, h_{6}\right\}}\right\}$

$J^{*}($ best school district $)=\left\{\frac{\text { near to market }}{\left\{h_{3}, h_{7}\right\}}, \frac{\text { with pool }}{\left\{h_{2}, h_{5}, h_{6}\right\}}, \frac{\text { wooden }}{\left\{h_{2}, h_{4}, h_{5}\right\}}, \frac{\text { beautiful }}{\left\{h_{1}, h_{6}\right\}}\right\}$.

3.19. Proposition. Let $\left[F^{*}, A\right],\left[G^{*}, B\right]$ and $\left[I^{*}, C\right]$ be three $T 2 S S$ defined over a common universe $U$. Then, for $\ell \in\{r, \varepsilon, r-\varepsilon, \varepsilon-r\}$ the following relations hold:

(i) $\left[F^{*}, A\right] \sqcup_{\ell}\left[F^{*}, A\right]=\left[F^{*}, A\right]$

(ii) $\left[F^{*}, A\right] \sqcap_{\ell}\left[F^{*}, A\right]=\left[F^{*}, A\right]$

(iii) $\left[F^{*}, A\right] \sqcup_{\ell}\left[G^{*}, B\right]=\left[G^{*}, B\right] \sqcup_{\ell}\left[F^{*}, A\right]$

(iv) $\left[F^{*}, A\right] \sqcap_{\ell}\left[G^{*}, B\right]=\left[G^{*}, B\right] \sqcap_{\ell}\left[F^{*}, A\right]$

$(v)\left(\left[F^{*}, A\right] \sqcup_{\ell}\left[G^{*}, B\right]\right) \sqcup_{\ell}\left[I^{*}, C\right]=\left[F^{*}, A\right] \sqcup_{\ell}\left(\left[G^{*}, B\right] \sqcup_{\ell}\left[I^{*}, C\right]\right)$

(vi) $\left(\left[F^{*}, A\right] \sqcap_{\ell}\left[G^{*}, B\right]\right) \sqcap_{\ell}\left[I^{*}, C\right]=\left[F^{*}, A\right] \sqcap_{\ell}\left(\left[G^{*}, B\right] \sqcap_{\ell}\left[I^{*}, C\right]\right)$

(vii) $\left[F^{*}, A\right] \sqcup_{\ell}\left(\left[G^{*}, B\right] \sqcap_{\ell}\left[I^{*}, C\right]\right)=\left(\left[F^{*}, A\right] \sqcup_{\ell}\left[G^{*}, B\right]\right) \sqcap_{\ell}\left(\left[F^{*}, A\right] \sqcup_{\ell}\left[I^{*}, C\right]\right)$

(viii) $\left[F^{*}, A\right] \sqcap_{\ell}\left(\left[G^{*}, B\right] \sqcup_{\ell}\left[I^{*}, C\right]\right)=\left(\left[F^{*}, A\right] \sqcap_{\ell}\left[G^{*}, B\right]\right) \sqcup_{\ell}\left(\left[F^{*}, A\right] \sqcap_{\ell}\left[I^{*}, C\right]\right)$

Proof. Proofs are straight-forward.

3.20. Proposition. Let $\left[F^{*}, A\right]$ be a $T 2 S S$ defined over a common universe $U$. Then, for $\ell \in\{r, \varepsilon, r-\varepsilon, \varepsilon-r\}$ the following relations hold:

(i) $\left[F^{*}, A\right] \sqcup_{\ell}\left[F^{*}, A\right]^{2}=W_{A}^{*}$

(ii) $\left[F^{*}, A\right] \sqcap_{\ell}\left[F^{*}, A\right]^{2}=\mathbb{N}_{A}^{*}$

Proof. It is obvious, therefore omitted. 
3.21. Proposition. Let $\left[F^{*}, A\right]$ and $\left[G^{*}, A\right]$ be two $T 2 S S$ defined over a common universe $U$. Then, the following relations hold:

(i) $\left[F^{*}, A\right] \sqcup_{r}\left[G^{*}, B\right] \sqsubseteq\left[F^{*}, A\right] \sqcup_{\varepsilon-r}\left[G^{*}, B\right] \sqsubseteq\left[F^{*}, A\right] \sqcup_{\varepsilon}\left[G^{*}, B\right]$

(ii) $\left[F^{*}, A\right] \sqcup_{r}\left[G^{*}, B\right] \sqsubseteq\left[F^{*}, A\right] \sqcup_{r-\varepsilon}\left[G^{*}, B\right] \sqsubseteq\left[F^{*}, A\right] \sqcup_{\varepsilon}\left[G^{*}, B\right]$

(iii) $\left[F^{*}, A\right] \sqcap_{r}\left[G^{*}, B\right] \sqsubseteq\left[F^{*}, A\right] \sqcap_{\varepsilon-r}\left[G^{*}, B\right] \sqsubseteq\left[F^{*}, A\right] \sqcap_{\varepsilon}\left[G^{*}, B\right]$

(iv) $\left[F^{*}, A\right] \sqcap_{r}\left[G^{*}, B\right] \sqsubseteq\left[F^{*}, A\right] \sqcap_{r-\varepsilon}\left[G^{*}, B\right] \sqsubseteq\left[F^{*}, A\right] \sqcap_{\varepsilon}\left[G^{*}, B\right]$

$(v)\left(\left[F^{*}, A\right] \sqcup_{r-\varepsilon}\left[G^{*}, B\right]\right) \sqcup_{\varepsilon}\left(\left[F^{*}, A\right] \sqcup_{\varepsilon-r}\left[G^{*}, B\right]\right)=\left[F^{*}, A\right] \sqcup_{\varepsilon}\left[G^{*}, B\right]$

(vi) $\left(\left[F^{*}, A\right] \sqcup_{r-\varepsilon}\left[G^{*}, B\right]\right) \sqcup_{r}\left(\left[F^{*}, A\right] \sqcup_{\varepsilon-r}\left[G^{*}, B\right]\right)=\left[F^{*}, A\right] \sqcup_{r}\left[G^{*}, B\right]$

(vii) $\left(\left[F^{*}, A\right] \sqcap_{r-\varepsilon}\left[G^{*}, B\right]\right) \sqcap_{\varepsilon}\left(\left[F^{*}, A\right] \sqcap_{\varepsilon-r}\left[G^{*}, B\right]\right)=\left[F^{*}, A\right] \sqcap_{\varepsilon}\left[G^{*}, B\right]$

(viii) $\left(\left[F^{*}, A\right] \sqcap_{r-\varepsilon}\left[G^{*}, B\right]\right) \sqcap_{r}\left(\left[F^{*}, A\right] \sqcap_{\varepsilon-r}\left[G^{*}, B\right]\right)=\left[F^{*}, A\right] \sqcap_{r}\left[G^{*}, B\right]$

Proof. Proofs are straight-forward.

Next, we apply concept of extension and restriction in difference of two $T 2 S S$.

3.22. Definition. Let $\left[F^{*}, A\right]$ and $\left[G^{*}, B\right]$ be two $T 2 S S$ over a universe $U$ such that $A \cap$ $B \neq \emptyset$. The restricted difference of $\left[F^{*}, A\right]$ and $\left[G^{*}, B\right]$ is denoted by $\left[F^{*}, A\right] \widehat{\Theta}_{r}\left[G^{*}, B\right]$ and is defined as $\left[F^{*}, A\right] \widehat{\Theta}_{r}\left[G^{*}, B\right]=\left[K^{*}, C\right]$ where $C=A \cap B$ and for all $c \in C$, $K^{*}(c)=F^{*}(c) \Theta_{r} G^{*}(c)$, where $K^{*}(c)=F^{*}(c) \Theta_{r} G^{*}(c)$ refers to restricted difference between the respective $T 1 S S$ corresponding to $F^{*}(c)$ and $G^{*}(c)$ respectively.

3.23. Definition. Let $\left[F^{*}, A\right]$ and $\left[G^{*}, B\right]$ be two $T 2 S S$ over a universe $U$ such that $A \cap B \neq \emptyset$. The restricted-extended difference of $\left[F^{*}, A\right]$ and $\left[G^{*}, B\right]$ is denoted by $\left[F^{*}, A\right] \widehat{\Theta}_{r-\varepsilon}\left[G^{*}, B\right]$ and is defined as $\left[F^{*}, A\right] \widehat{\Theta}_{r-\varepsilon}\left[G^{*}, B\right]=\left[K^{*}, C\right]$ where $C=A \cap B$ and for all $c \in C, K^{*}(c)=F^{*}(c) \ominus_{\varepsilon} G^{*}(c)$, where $K^{*}(c)=F^{*}(c) \ominus_{\varepsilon} G^{*}(c)$ refers to extended difference between the respective $T 1 S S$ corresponding to $F^{*}(c)$ and $G^{*}(c)$ respectively.

3.24. Definition. Let $\left[F^{*}, A\right]$ and $\left[G^{*}, B\right]$ be two $T 2 S S$ over a universe $U$. The extended difference of $\left[F^{*}, A\right]$ and $\left[G^{*}, B\right]$ is denoted by $\left[F^{*}, A\right] \widehat{\Theta}_{\varepsilon}\left[G^{*}, B\right]$ and is defined as $\left[F^{*}, A\right] \widehat{\ominus}_{\varepsilon}\left[G^{*}, B\right]=\left[K^{*}, C\right]$ where $C=A \cup B$ and for all $c \in C$,

$$
H^{*}(c)=\left\{\begin{array}{cc}
F^{*}(c), & \text { if } c \in A-B \\
G^{*}(c), & \text { if } c \in B-A \\
F^{*}(c) \Theta_{\varepsilon} G^{*}(c), & \text { if } c \in A \cap B
\end{array}\right.
$$

where $F^{*}(c) \ominus_{\varepsilon} G^{*}(c)$ refers to extended difference between the respective $T 1 S S$ corresponding to $F^{*}(c)$ and $G^{*}(c)$ respectively.

3.25. Definition. Let $\left[F^{*}, A\right]$ and $\left[G^{*}, B\right]$ be two $T 2 S S$ over a universe $U$. The extendedrestricted difference of $\left[F^{*}, A\right]$ and $\left[G^{*}, B\right]$ is denoted by $\left[F^{*}, A\right] \ominus_{\varepsilon-r}\left[G^{*}, B\right]$ and is defined as $\left[F^{*}, A\right] \ominus_{\varepsilon-r}\left[G^{*}, B\right]=\left[K^{*}, C\right]$ where $C=A \cup B$ and for all $c \in C$,

$$
H^{*}(c)=\left\{\begin{array}{cc}
F^{*}(c), & \text { if } c \in A-B \\
G^{*}(c), & \text { if } c \in B-A \\
F^{*}(c) \Theta_{r} G^{*}(c), & \text { if } c \in A \cap B
\end{array}\right.
$$

where $F^{*}(c) \Theta_{r} G^{*}(c)$ refers to restricted difference between the respective $T 1 S S$ corresponding to $F^{*}(c)$ and $G^{*}(c)$ respectively. 
3.26. Proposition. Let $\left[F^{*}, A\right]$ be a $T 2 S S$ defined over a common universe $U$. Then, for $\ell \in\{r, \varepsilon, r-\varepsilon, \varepsilon-r\}$ the following relations hold:
(i) $\left[F^{*}, A\right] \widehat{\Theta}_{\ell} \mathbb{N}_{A}^{*}=\left[F^{*}, A\right] \widehat{\Theta}_{\ell} \mathbb{N}_{E}^{*}=\left[F^{*}, A\right]$
(ii) $\left[F^{*}, A\right] \widehat{\Theta}_{\ell}\left[F^{*}, A\right]=\mathbb{N}_{(U, E)}^{*}$
(iii) $W_{A}^{*} \widehat{\Theta}_{\ell}\left[F^{*}, A\right]=\left[F^{*}, A\right]^{2}$
(iv) $W_{E}^{*} \widehat{\Theta}_{\ell}\left[F^{*}, A\right]=\left[F^{*}, A\right]^{2}$

Proof. Proofs are straight-forward.

3.27. Proposition. Let $\left[F^{*}, A\right]$ and $\left[G^{*}, B\right]$ be two $T 2 S S$ defined over a common universe $U$. Then, the following relations hold:

(i) $\left[F^{*}, A\right] \widehat{\Theta}_{r}\left[G^{*}, B\right] \sqsubseteq\left[F^{*}, A\right] \widehat{\Theta}_{\varepsilon-r}\left[G^{*}, B\right] \sqsubseteq\left[F^{*}, A\right] \widehat{\Theta}_{\varepsilon}\left[G^{*}, B\right]$

(ii) $\left[F^{*}, A\right] \widehat{\Theta}_{r}\left[G^{*}, B\right] \sqsubseteq\left[F^{*}, A\right] \widehat{\Theta}_{r-\varepsilon}\left[G^{*}, B\right] \sqsubseteq\left[F^{*}, A\right] \widehat{\Theta}_{\varepsilon}\left[G^{*}, B\right]$

Proof. Proofs are straight-forward.

3.28. Definition. The restricted complement of a $T 2 S S\left[F^{*}, A\right]$ is denoted as $\left[F^{*}, A\right]^{2 r}=$ $\left[\left(F^{*}\right)^{2 r}, A\right]=\omega_{A}^{*} \widehat{\Theta}_{r}\left[F^{*}, A\right]$ and is defined as $\left(F^{*}\right)^{2_{r}}(\alpha)=U^{*} \Theta_{r} F^{*}(\alpha)$ for all $\alpha \in A$, where $\omega_{A}^{*}=\left[U^{*}, A\right]$ is a complete $T 2 S S$ of $A$ over $U$.

3.29. Lemma. Let $\left[F^{*}, A\right]$ be a $T 2 S S$ over a universe $U$. Then $\left[F^{*}, A\right]^{2 r}=\left[F^{*}, A\right]^{2}$.

Proof. Proof is straightforward therefore omitted.

By Lemma 3.29, we will use the Definition 3.28, for complements in rest of paper and use the symbol $\left[F^{*}, A\right]^{2}$ in place of $\left[F^{*}, A\right]^{2 r}$ for complement of $\left[F^{*}, A\right]$.

\section{De Morgan's laws in Type-2 soft set theory}

In this section, we show that the following De Morgan's laws type of results holds in $T 2 S S$ theory for the newly defined the restricted union, the extended union, the restricted intersection, the extended intersection, the restricted-extended union, the extendedrestricted union, the restricted-extended intersection and the extended-restricted intersection.

4.1. Theorem. Let $\left[F^{*}, A\right]$ and $\left[G^{*}, B\right]$ be two $T 2 S S$ over a common universe $U$ such that $A \cap B \neq \emptyset$. Then,

(i) $\left(\left[F^{*}, A\right] \sqcup_{r}\left[G^{*}, B\right]\right)^{2}=\left[F^{*}, A\right]^{2} \sqcap_{r}\left[G^{*}, B\right]^{2}$

(ii) $\left(\left[F^{*}, A\right] \sqcap_{r}\left[G^{*}, B\right]\right)^{2}=\left[F^{*}, A\right]^{2} \sqcup_{r}\left[G^{*}, B\right]^{2}$

Proof. (i) Let $\left[F^{*}, A\right] \sqcup_{r}\left[G^{*}, B\right]=\left[H^{*}, A \cap B\right]$ such that $C=A \cap B \neq \emptyset$. Then, $H^{*}(\alpha)=$ $F^{*}(\alpha) \widetilde{\cup}_{r} G^{*}(\alpha)$ for all $\alpha \in C$. Since $\left(\left[F^{*}, A\right] \sqcup_{r}\left[G^{*}, B\right]\right)^{2}=\left[H^{*}, A \cap B\right]^{2}$, by Lemma 3.29, we have $\left(H^{*}\right)^{2}(\alpha)=U^{*} \Theta_{r} F^{*}(\alpha) \widetilde{\cup}_{r} G^{*}(\alpha)$ for all $\alpha \in C$. Thus,

$$
\begin{aligned}
\left(H^{*}\right)^{2}(\alpha) & =\left\{\frac{\gamma}{U-\left(F_{\alpha}(\gamma)\right) \cup_{r}\left(G_{\alpha}(\gamma)\right)} \text { if } \gamma \in L_{\alpha} \cap M_{\alpha}\right. \\
& =\left\{\frac{\gamma}{\left(U-F_{\alpha}(\gamma)\right) \cap_{r}\left(U-G_{\alpha}(\gamma)\right)} \text { if } \gamma \in L_{\alpha} \cap M_{\alpha}\right. \\
& =\left\{\frac{\gamma}{\left(F_{\alpha}^{2}(\gamma)\right) \cap_{r}\left(G_{\alpha}^{2}(\gamma)\right)} \text { if } \gamma \in L_{\alpha} \cap M_{\alpha}\right.
\end{aligned}
$$

where $L_{\alpha}$ and $M_{\alpha}$ are the sets of underlying parameters of $T 1 S S\left(F_{\alpha}, L_{\alpha}\right)$ and $\left(G_{\alpha}, M_{\alpha}\right)$ corresponding to $F^{*}(\alpha)$ and $G^{*}(\alpha)$ respectively. 
On the other hand, $\left[F^{*}, A\right]^{2} \sqcap_{r}\left[G^{*}, B\right]^{2}=\left[\left(F^{*}\right)^{2}, A\right] \sqcap_{r}\left[\left(G^{*}\right)^{2}, B\right]=\left[K^{*}, A \cap B\right]$, where $C=A \cap B$. Since $K^{*}(\alpha)=\left(F^{*}\right)^{2}(\alpha) \widetilde{\cap}_{r}\left(G^{*}\right)^{2}(\alpha)$ for all $\alpha \in A \cap B$. Thus,

$$
K^{*}(\alpha)=\left\{\frac{\gamma}{\left(F_{\alpha}^{2}(\gamma)\right) \cap_{r}\left(G_{\alpha}^{2}(\gamma)\right)} \text { if } \gamma \in L_{\alpha}^{\prime} \cap M_{\alpha}^{\prime}\right.
$$

where $L_{\alpha}^{\prime}$ and $M_{\alpha}^{\prime}$ are the sets of underlying parameters of $T 1 S S\left(F_{\alpha}, L_{\alpha}\right)$ and $\left(G_{\alpha}, M_{\alpha}\right)$ corresponding to $F^{*}(\alpha)$ and $G^{*}(\alpha)$ respectively.

Since $\left(H^{*}\right)^{2}$ and $K^{*}$ are indeed the same set-valued mapping, we conclude that $\left(\left[F^{*}, A\right] \sqcup_{r}\left[G^{*}, B\right]\right)^{2}=\left[F^{*}, A\right]^{2} \sqcap_{r}\left[G^{*}, B\right]^{2}$ as required. Similarly, we can prove $(i i)$.

4.2. Theorem. Let $\left[F^{*}, A\right]$ and $\left[G^{*}, B\right]$ be two $T 2 S S$ over a common universe $U$. Then,

(i) $\left(\left[F^{*}, A\right] \sqcup_{\epsilon}\left[G^{*}, B\right]\right)^{2}=\left[F^{*}, A\right]^{2} \sqcap_{\varepsilon}\left[G^{*}, B\right]^{2}$

(ii) $\left(\left[F^{*}, A\right] \sqcap_{\varepsilon}\left[G^{*}, B\right]\right)^{2}=\left[F^{*}, A\right]^{2} \sqcup_{\epsilon}\left[G^{*}, B\right]^{2}$

Proof. (i) Let $\left[F^{*}, A\right] \sqcup_{\varepsilon}\left[G^{*}, B\right]=\left[H^{*}, C\right]$ such that $C=A \cup B$. For all $\alpha \in C$, by definition, we have

$$
H^{*}(\alpha)= \begin{cases}F^{*}(\alpha), & \text { if } \alpha \in A-B, \\ G^{*}(\alpha), & \text { if } \alpha \in B-A, \\ F^{*}(\alpha) \widetilde{\cup}_{\varepsilon} G^{*}(\alpha), & \text { if } \alpha \in A \cap B .\end{cases}
$$

and

$$
\left(H^{*}\right)^{2}(\alpha)=\left\{\begin{array}{lr}
U^{*} \ominus_{r} F^{*}(\alpha), & \text { if } \alpha \in A-B, \\
U^{*} \ominus_{r} G^{*}(\alpha), & \text { if } \alpha \in B-A, \\
U^{*} \ominus_{r} F^{*}(\alpha) \widetilde{\cup}_{\varepsilon} G^{*}(\alpha), & \text { if } \alpha \in A \cap B .
\end{array}\right.
$$

It may be mentioned here that,

$$
\begin{aligned}
& U^{*} \Theta_{r} F^{*}(\alpha)=\left\{\frac{\beta}{U-F_{\alpha}(\beta)}=\frac{\beta}{F_{\alpha}^{2}(\beta)} \text { if } \beta \in L_{\alpha}, \alpha \in A-B,\right. \\
& U^{*} \ominus_{r} G^{*}(\alpha)=\left\{\frac{\delta}{U-G_{\alpha}(\delta)}=\frac{\delta}{G_{\alpha}^{2}(\delta)} \text { if } \delta \in M_{\alpha}, \alpha \in B-A\right.
\end{aligned}
$$

where $L_{\alpha}$ and $M_{\alpha}$ are the sets of underlying parameters of $T 1 S S\left(F_{\alpha}, L_{\alpha}\right)$ and $\left(G_{\alpha}, M_{\alpha}\right)$ corresponding to $F^{*}(\alpha)$ and $G^{*}(\alpha)$ respectively.

And

$$
\begin{aligned}
U^{*} \Theta_{r} F^{*}(\alpha) \widetilde{\cup}_{\varepsilon} G^{*}(\alpha) & =\left\{\begin{array}{lc}
\frac{\gamma}{U-F_{\alpha}(\gamma)}, & \text { if } \gamma \in L_{\alpha}^{\prime}, \\
\frac{\gamma}{U-G_{\alpha}(\gamma)}, & \text { if } \gamma \in M_{\alpha}^{\prime}, \\
\frac{\gamma}{U-F_{\alpha}(\gamma) \cup G_{\alpha}(\gamma)}, & \text { if } \gamma \in L_{\alpha}^{\prime} \cap M_{\alpha}^{\prime} .
\end{array}\right. \\
& = \begin{cases}\frac{\gamma}{F_{\alpha}^{2}(\gamma)}, & \text { if } \gamma \in L_{\alpha}^{\prime}, \\
\frac{\gamma}{G_{\alpha}^{2}(\gamma)}, & \text { if } \gamma \in M_{\alpha}^{\prime}, \\
\frac{\gamma}{F_{\alpha}^{2}(\gamma) \cap G_{\alpha}^{2}(\gamma)}, & \text { if } \gamma \in L_{\alpha}^{\prime} \cap M_{\alpha}^{\prime} .\end{cases}
\end{aligned}
$$

for all $\alpha \in A \cap B$, where $L_{\alpha}^{\prime}$ and $M_{\alpha}^{\prime}$ are the sets of underlying parameters of $T 1 S S$ $\left(F_{\alpha}, L_{\alpha}\right)$ and $\left(G_{\alpha}, M_{\alpha}\right)$ corresponding to $F^{*}(\alpha)$ and $G^{*}(\alpha)$ respectively.

On the other hand, $\left[F^{*}, A\right]^{2} \sqcap_{\varepsilon}\left[G^{*}, B\right]^{2}=\left[K^{*}, C\right]$ such that $C=A \cap B$. For all $\alpha \in C$, by definition, we have 
$K^{*}(\alpha)=\left\{\begin{array}{cl}\left(F^{*}\right)^{2}(\alpha), & \text { if } \alpha \in A-B, \\ \left(G^{*}\right)^{2}(\alpha), & \text { if } \alpha \in B-A, \\ \left(\left(F^{*}\right)^{2}(\alpha)\right) \widetilde{\cap}_{\varepsilon}\left(\left(G^{*}\right)^{2}(\alpha)\right), & \text { if } \alpha \in A \cap B .\end{array}\right.$

It may be mentioned here that,

$$
\begin{aligned}
& \left(F^{*}\right)^{2}(\alpha)=\left\{\frac{\beta}{F_{\alpha}^{2}(\beta)} \text { if } \beta \in L_{\alpha}, \forall \alpha \in A-B,\right. \\
& \left(G^{*}\right)^{2}(\alpha)=\left\{\frac{\delta}{G_{\alpha}^{2}(\delta)} \text { if } \delta \in M_{\alpha}, \forall \alpha \in B-A\right.
\end{aligned}
$$

where $L_{\alpha}$ and $M_{\alpha}$ are the sets of underlying parameters of $T 1 S S\left(F_{\alpha}, L_{\alpha}\right)$ and $\left(G_{\alpha}, M_{\alpha}\right)$ corresponding to $F^{*}(\alpha)$ and $G^{*}(\alpha)$ respectively.

And

$$
\left(\left(F^{*}\right)^{2}(\alpha)\right) \widetilde{\cap}_{\varepsilon}\left(\left(G^{*}\right)^{2}(\alpha)\right)=\left\{\begin{array}{cc}
\frac{\gamma}{F_{\alpha}^{2}(\gamma)}, & \text { if } \gamma \in L_{\alpha}^{\prime}, \\
\frac{\gamma}{G_{\alpha}^{2}(\gamma)}, & \text { if } \gamma \in M_{\alpha}^{\prime}, \\
\frac{\gamma}{F_{\alpha}^{2}(\gamma) \cap G_{\alpha}^{2}(\gamma)}, & \text { if } \gamma \in L_{\alpha}^{\prime} \cap M_{\alpha}^{\prime} .
\end{array}\right.
$$

for all $\alpha \in A \cap B$, where $L_{\alpha}^{\prime}$ and $M_{\alpha}^{\prime}$ are the sets of underlying parameters of $T 1 S S$ $\left(F_{\alpha}, L_{\alpha}^{\prime}\right)$ and $\left(G_{\alpha}, M_{\alpha}^{\prime}\right)$ corresponding to $F^{*}(\alpha)$ and $G^{*}(\alpha)$ respectively.

Since $\left(H^{*}\right)^{2}$ and $K^{*}$ are indeed the same set-valued mapping, we conclude that $\left(\left[F^{*}, A\right] \sqcup_{\varepsilon}\left[G^{*}, B\right]\right)^{2}=\left[F^{*}, A\right]^{2} \Pi_{\varepsilon}\left[G^{*}, B\right]^{2}$ as required. Similarly, we can prove $(i i)$.

4.3. Theorem. Let $\left[F^{*}, A\right]$ and $\left[G^{*}, B\right]$ be two $T 2 S S$ over same universe $U$ such that $A \cap B \neq \emptyset$. Then,

(i) $\left(\left[F^{*}, A\right] \sqcup_{r-\epsilon}\left[G^{*}, B\right]\right)^{2}=\left[F^{*}, A\right]^{2} \sqcap_{r-\varepsilon}\left[G^{*}, B\right]^{2}$

(ii) $\left(\left[F^{*}, A\right] \sqcap_{r-\varepsilon}\left[G^{*}, B\right]\right)^{2}=\left[F^{*}, A\right]^{2} \sqcup_{r-\varepsilon}\left[G^{*}, B\right]^{2}$

Proof. (i) Let $\left[F^{*}, A\right] \sqcup_{r-\varepsilon}\left[G^{*}, B\right]=\left[H^{*}, A \cap B\right]$ such that $C=A \cap B \neq \emptyset$. Then, $H^{*}(\alpha)=F^{*}(\alpha) \widetilde{\cup}_{\varepsilon} G^{*}(\alpha)$ for all $\alpha \in C$. Since $\left(\left[F^{*}, A\right] \sqcup_{r-\varepsilon}\left[G^{*}, B\right]\right)^{2}=\left[H^{*}, A \cap B\right]^{2}$, by Lemma 3.29, we have

$$
\begin{aligned}
\left(H^{*}\right)^{2}(\alpha) & =U^{*} \Theta_{r} F^{*}(\alpha) \widetilde{\cup}_{\varepsilon} G^{*}(\alpha) \\
& =\left\{\begin{array}{lr}
\frac{\gamma}{U-F_{\alpha}(\gamma)}, & \text { if } \gamma \in L_{\alpha}^{\prime}, \\
\frac{\gamma}{U-G_{\alpha}(\gamma)}, & \text { if } \gamma \in M_{\alpha}^{\prime}, \\
\frac{\gamma}{U-F_{\alpha}(\gamma) \cup G_{\alpha}(\gamma)}, & \text { if } \gamma \in L_{\alpha}^{\prime} \cap M_{\alpha}^{\prime} .
\end{array}\right. \\
& =\left\{\begin{array}{lr}
\frac{\gamma}{F_{\alpha}^{2}(\gamma)}, & \text { if } \gamma \in L_{\alpha}^{\prime}, \\
\frac{\gamma}{G_{\alpha}^{2}(\gamma)}, & \text { if } \gamma \in M_{\alpha}^{\prime}, \\
\frac{\gamma}{F_{\alpha}^{2}(\gamma) \cap G_{\alpha}^{2}(\gamma)}, & \text { if } \gamma \in L_{\alpha}^{\prime} \cap M_{\alpha}^{\prime} .
\end{array}\right.
\end{aligned}
$$

for all $\alpha \in A \cap B$, where $L_{\alpha}^{\prime}$ and $M_{\alpha}^{\prime}$ are the sets of underlying parameters of $T 1 S S$ $\left(F_{\alpha}, L_{\alpha}\right)$ and $\left(G_{\alpha}, M_{\alpha}\right)$ corresponding to $F^{*}(\alpha)$ and $G^{*}(\alpha)$ respectively.

On the other hand, $\left[F^{*}, A\right]^{2} \sqcap_{r-\varepsilon}\left[G^{*}, B\right]^{2}=\left[\left(F^{*}\right)^{2}, A\right] \sqcap_{r-\varepsilon}\left[\left(G^{*}\right)^{2}, B\right]=\left[K^{*}, A \cap B\right]$, where $C=A \cap B$. Then $K^{*}(\alpha)=\left(F^{*}\right)^{2}(\alpha) \widetilde{\cap}_{\varepsilon}\left(G^{*}\right)^{2}(\alpha)$. It may be mentioned here that, 


$$
K^{*}(\alpha)=\left\{\begin{array}{cc}
\frac{\gamma}{F_{\alpha}^{2}(\gamma)}, & \text { if } \gamma \in L_{\alpha}^{\prime}, \\
\frac{\gamma}{G_{\alpha}^{2}(\gamma)}, & \text { if } \gamma \in M_{\alpha}^{\prime}, \\
\frac{\gamma}{F_{\alpha}^{2}(\gamma) \cap G_{\alpha}^{2}(\gamma)}, & \text { if } \gamma \in L_{\alpha}^{\prime} \cap M_{\alpha}^{\prime} .
\end{array}\right.
$$

for all $\alpha \in A \cap B$, where $L_{\alpha}^{\prime}$ and $M_{\alpha}^{\prime}$ are the sets of underlying parameters of $T 1 S S$ $\left(F_{\alpha}, L_{\alpha}^{\prime}\right)$ and $\left(G_{\alpha}, M_{\alpha}^{\prime}\right)$ corresponding to $F^{*}(\alpha)$ and $G^{*}(\alpha)$ respectively.

Since $\left(H^{*}\right)^{2}$ and $K^{*}$ are indeed the same set-valued mapping, we conclude that $\left(\left[F^{*}, A\right] \sqcup_{r-\epsilon}\left[G^{*}, B\right]\right)^{2}=\left[F^{*}, A\right]^{2} \sqcap_{r-\varepsilon}\left[G^{*}, B\right]^{2}$ as required. Similarly, we can prove (ii).

4.4. Theorem. Let $\left[F^{*}, A\right]$ and $\left[G^{*}, B\right]$ be two $T 2 S S$ over same universe $U$. Then,

(i) $\left(\left[F^{*}, A\right] \sqcup_{\varepsilon-r}\left[G^{*}, B\right]\right)^{2}=\left[F^{*}, A\right]^{2} \sqcap_{\varepsilon-r}\left[G^{*}, B\right]^{2}$

(ii) $\left(\left[F^{*}, A\right] \sqcap_{\varepsilon-r}\left[G^{*}, B\right]\right)^{2}=\left[F^{*}, A\right]^{2} \sqcup_{\varepsilon-r}\left[G^{*}, B\right]^{2}$

Proof. (i) Let $\left[F^{*}, A\right] \sqcup_{\varepsilon-r}\left[G^{*}, B\right]=\left[H^{*}, A \cap B\right]$ such that $C=A \cap B \neq \emptyset$. For all $\alpha \in C$, by definition, we have

$$
H^{*}(\alpha)= \begin{cases}F^{*}(\alpha), & \text { if } \alpha \in A-B \\ G^{*}(\alpha), & \text { if } \alpha \in B-A \\ F^{*}(\alpha) \widetilde{\cup}_{r} G^{*}(\alpha), & \text { if } \alpha \in A \cap B\end{cases}
$$

and

$$
\left(H^{*}\right)^{2}(\alpha)=\left\{\begin{array}{lr}
U^{*} \Theta_{r} F^{*}(\alpha), & \text { if } \alpha \in A-B \\
U^{*} \Theta_{r} G^{*}(\alpha), & \text { if } \alpha \in B-A \\
U^{*} \Theta_{r} F^{*}(\alpha) \widetilde{\cup}_{r} G^{*}(\alpha), & \text { if } \alpha \in A \cap B
\end{array}\right.
$$

It may be mention that,

$$
\begin{aligned}
& U^{*} \ominus_{r} F^{*}(\alpha)=\left\{\frac{\beta}{U-F_{\alpha}(\beta)}=\frac{\beta}{F_{\alpha}^{2}(\beta)} \text { if } \quad \beta \in L_{\alpha}, \forall \alpha \in A-B\right. \\
& U^{*} \ominus_{r} G^{*}(\alpha)=\left\{\frac{\delta}{U-G_{\alpha}(\delta)}=\frac{\delta}{G_{\alpha}^{2}(\delta)} \text { if } \quad \delta \in M_{\alpha}, \forall \alpha \in B-A\right.
\end{aligned}
$$

where $L_{\alpha}$ and $M_{\alpha}$ are the sets of underlying parameters of $T 1 S S\left(F_{\alpha}, L_{\alpha}\right)$ and $\left(G_{\alpha}, M_{\alpha}\right)$ corresponding to $F^{*}(\alpha)$ and $G^{*}(\alpha)$ respectively. And

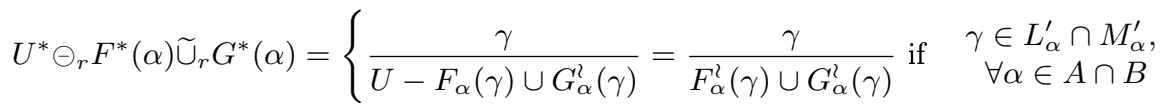

where $L_{\alpha}^{\prime}$ and $M_{\alpha}^{\prime}$ are the sets of underlying parameters of $T 1 S S\left(F_{\alpha}, L_{\alpha}\right)$ and $\left(G_{\alpha}, M_{\alpha}\right)$ corresponding to $F^{*}(\alpha)$ and $G^{*}(\alpha)$ respectively.

On the other hand, $\left[F^{*}, A\right]^{2} \Pi_{\varepsilon-r}\left[G^{*}, B\right]^{2}=\left[K^{*}, C\right]$ such that $C=A \cup B$. For all $\alpha \in C$, by definition, we have

$$
K^{*}(\alpha)=\left\{\begin{array}{cc}
\left(F^{*}\right)^{2}(\alpha), & \text { if } \alpha \in A-B, \\
\left(G^{*}\right)^{2}(\alpha), & \text { if } \alpha \in B-A, \\
\left(\left(F^{*}\right)^{2}(\alpha)\right) \widetilde{\cap}_{r}\left(\left(G^{*}\right)^{2}(\alpha)\right), & \text { if } \alpha \in A \cap B .
\end{array}\right.
$$

It may be mention that

$$
\begin{aligned}
& \left(F^{*}\right)^{2}(\alpha)=\left\{\frac{\beta}{F_{\alpha}^{2}(\beta)} \text { if } \beta \in L_{\alpha}, \forall \alpha \in A-B\right. \\
& \left(G^{*}\right)^{2}(\alpha)=\left\{\frac{\delta}{G_{\alpha}^{2}(\delta)} \text { if } \delta \in M_{\alpha}, \forall \alpha \in B-A\right.
\end{aligned}
$$


where $L_{\alpha}$ and $M_{\alpha}$ are the sets of underlying parameters of $T 1 S S\left(F_{\alpha}, L_{\alpha}\right)$ and $\left(G_{\alpha}, M_{\alpha}\right)$ corresponding to $F^{*}(\alpha)$ and $G^{*}(\alpha)$ respectively. And

$\left(\left(F^{*}\right)^{2}(\alpha)\right) \widetilde{\cap}_{r}\left(\left(G^{*}\right)^{2}(\alpha)\right)=\left\{\frac{\gamma}{F_{\alpha}^{2}(\gamma) \cap G_{\alpha}^{2}(\gamma)}\right.$ if $\gamma \in L_{\alpha}^{\prime} \cap M_{\alpha}^{\prime} \forall \alpha \in A \cap B$

for all $\alpha \in A \cap B$, where $L_{\alpha}^{\prime}$ and $M_{\alpha}^{\prime}$ are the sets of underlying parameters of $T 1 S S$ $\left(F_{\alpha}, L_{\alpha}^{\prime}\right)$ and $\left(G_{\alpha}, M_{\alpha}^{\prime}\right)$ corresponding to $F^{*}(\alpha)$ and $G^{*}(\alpha)$ respectively.

Since $\left(H^{*}\right)^{2}$ and $K^{*}$ are indeed the same set-valued mapping, we conclude that $\left(\left[F^{*}, A\right] \sqcup_{r-\epsilon}\left[G^{*}, B\right]\right)^{2}=\left[F^{*}, A\right]^{2} \sqcap_{r-\varepsilon}\left[G^{*}, B\right]^{2}$ as required. Similarly, we can prove (ii).

In order to explain above theorems an example is given in the following.

4.5. Example. A car company is desirable to select the unsuitable robots on different robot attributes. Assume a set $U$ of seven robots under consideration, which is denoted by $U=\left\{h_{1}, h_{2}, h_{3}, h_{4}, h_{5}, h_{6}, h_{7}\right\}$ and $E$ is a parameter set, where

$$
\begin{aligned}
E & =\left\{e_{1}, e_{2}, e_{3}, e_{4}, e_{5}, e_{6}, e_{7}, e_{8}, e_{9}, e_{10}\right\} \\
& =\left\{\begin{array}{c}
\text { aluminum material, medium size, smallweight, high cost, with a good } \\
\text { driving system, high load capacity, high repeatability, } \\
\text { high speed, medium memory capacity, high degree of freedom }
\end{array}\right\} .
\end{aligned}
$$

This company has two decision makers from consultancy departments: $F^{*}$ is from the production department, $G^{*}$ is from the engineering department. They make observations on provided primary attributes: aluminum material, medium size, small weight, high cost.

The $F^{*}$ considers a set of parameters

$A=\{$ aluminum material, small weight, high cost $\}$

and the $G^{*}$ considers a set of parameters

$B=\{$ medium size, small weight, high cost $\}$ such that $A, B \subseteq E$. They have observations (type-2 soft sets) $\left[F^{*}, A\right]$ and $\left[G^{*}, B\right]$ as follows

$$
\begin{aligned}
& F^{*}(\text { aluminum material })=\left\{\frac{\text { high speed }}{\left\{h_{2}, h_{4}, h_{5}\right\}}, \frac{\text { high degree of freedom }}{\left\{h_{6}, h_{7}\right\}}\right\} \\
& F^{*}(\text { small weight })=\left\{\frac{\text { medium memory capacity }}{\left\{h_{3}, h_{5}\right\}}, \frac{\text { high repeatability }}{\left\{h_{1}, h_{3}, h_{7}\right\}}, \frac{\text { high degree of freedom }}{\left\{h_{1}, h_{4}, h_{6}\right\}}\right\} \\
& F^{*}(\text { high cost })=\left\{\frac{\text { high speed }}{\left\{h_{1}, h_{3}, h_{5}\right\}}, \frac{\text { high repeatability }}{\left\{h_{1}, h_{7}\right\}}, \frac{\text { high load capacity }}{\left\{h_{1}, h_{2}, h_{7}\right\}}\right\} \\
& \text { and } \\
& G^{*}(\text { medium size })=\left\{\frac{\text { high load capacity }}{\left\{h_{3}, h_{4}, h_{6}\right\}}, \frac{\text { high degree of freedom }}{\left\{h_{2}, h_{5}, h_{7}\right\}}\right\} \\
& G^{*}(\text { small weight })=\left\{\begin{array}{c}
\frac{\text { medium memory capacity }}{\left\{h_{3}, h_{5}, h_{7}\right\}}, \frac{\text { high speed }}{\left\{h_{2}, h_{3}, h_{5}\right\}}, \\
\frac{\text { high degree of freedom }}{\left\{h_{2}, h_{4}, h_{6}\right\}}
\end{array}\right\} \\
& G^{*}(\text { high cost })=\left\{\frac{\text { high repeatability }}{\left\{h_{2}, h_{3}, h_{7}\right\}}, \frac{\text { high load capacity }}{\left\{h_{2}, h_{4}, h_{6}\right\}}\right\}
\end{aligned}
$$

Note that the set of underlying parameters of decision makers is

$\left\{\begin{array}{c}\text { high load capacity, high repeatability, high speed, high degree of freedom, } \\ \text { medium memory capacity }\end{array}\right\}$. 
Now this company interesting to obtain most unsuitable robots based on above observations of decision makers. Then company may consider one of the two problems $\left(\Gamma_{1}\right)$ and $\left(\Gamma_{2}\right)$ on primary attributes as in the following.

$\left(\Gamma_{1}\right)$ In order to get most unsuitable robots based on these observations company interesting on results of each primary attributes: aluminum material, medium size, small weight, high cost.

(i) Suppose company looks at both complete set of primary parameters and the complete sets underlying parameters of decision makers. The following two methods to obtain the results

$\left(p_{1}\right)$ In first method company combine both observations by using extended union and then takes the complement of it. Then $\left[F^{*}, A\right] \sqcup_{\varepsilon}\left[G^{*}, B\right]=\left[I^{*}, C\right]$, where $C=A \cup B$. For all $\alpha \in C,\left[I^{*}, C\right]$ is as the following,

$I^{*}($ aluminum material $)=\left\{\frac{\text { high speed }}{\left\{h_{2}, h_{4}, h_{5}\right\}}, \frac{\text { high degree of freedom }}{\left\{h_{6}, h_{7}\right\}}\right\}$

$I^{*}($ medium size $)=\left\{\frac{\text { high load capacity }}{\left\{h_{3}, h_{4}, h_{6}\right\}}, \frac{\text { high degree of freedom }}{\left\{h_{2}, h_{5}, h_{7}\right\}}\right\}$

$I^{*}($ small weight $)=\left\{\begin{array}{c}\frac{\text { medium memory capacity }}{\left\{h_{3}, h_{5}, h_{7}\right\}}, \frac{\text { high speed }}{\left\{h_{2}, h_{3}, h_{5}\right\}}, \\ \frac{\text { high degree of freedom }}{\left\{h_{1}, h_{2}, h_{4}, h_{6}\right\}}, \frac{\text { high repeatability }}{\left\{h_{1}, h_{3}, h_{7}\right\}}\end{array}\right\}$

$I^{*}($ high cost $)=\left\{\frac{\text { high repeatability }}{\left\{h_{1}, h_{2}, h_{3}, h_{7}\right\}}, \frac{\text { high speed }}{\left\{h_{1}, h_{3}, h_{5}\right\}}, \frac{\text { high load capacity }}{\left\{h_{1}, h_{2}, h_{4}, h_{6}, h_{7}\right\}}\right\}$.

Then

$\left(I^{*}\right)^{2}$ (aluminum material $)=\left\{\frac{\text { high speed }}{\left\{h_{1}, h_{3}, h_{6}, h_{7}\right\}}, \frac{\text { high of freedom }}{\left\{h_{1}, h_{2}, h_{3}, h_{4}, h_{5}\right\}}\right\}$

$\left(I^{*}\right)^{2}($ medium size $)=\left\{\frac{\text { high load capacity }}{\left\{h_{1}, h_{2}, h_{5}, h_{7}\right\}}, \frac{\text { high degree of freedom }}{\left\{h_{1}, h_{3}, h_{4}, h_{6}\right\}}\right\}$

$\left(I^{*}\right)^{2}($ small weight $)=\left\{\begin{array}{c}\frac{\text { medium memory capacity }}{\left\{h_{1}, h_{2}, h_{4}, h_{6}\right\}}, \frac{\text { high speed }}{\left\{h_{1}, h_{4}, h_{6}, h_{7}\right\}}, \\ \frac{\text { high degree of freedom }}{\left\{h_{3}, h_{5}, h_{7}\right\}}, \frac{\text { high repeatability }}{\left\{h_{2}, h_{4}, h_{5}, h_{6}\right\}}\end{array}\right\}$

$\left(I^{*}\right)^{2}($ high cost $)=\left\{\begin{array}{c}\frac{\text { high repeatability }}{\left\{h_{4}, h_{5}, h_{6}\right\}}, \frac{\text { high speed }}{\left\{h_{2}, h_{4}, h_{6}, h_{7}\right\}}, \\ \frac{\text { high load capacity }}{\left\{h_{3}, h_{5}\right\}}\end{array}\right\}$

These are the most unsuitable robots on the complete set primary attributes and on the complete sets of underlying parameters corresponding to primary attributes.

$\left(q_{1}\right)$ In this method company first takes complements of both observations $F^{*}$ and $G^{*}$ and obtain unsuitable robots from both of them. Then by using extended intersection company gets the result.

$\left(F^{*}\right)^{2}$ (aluminum material $)=\left\{\frac{\text { high speed }}{\left\{h_{1}, h_{3}, h_{6}, h_{7}\right\}}, \frac{\text { high degree of freedom }}{\left\{h_{1}, h_{2}, h_{3}, h_{4}, h_{5}\right\}}\right\}$

$\left(F^{*}\right)^{2}($ small weight $)=\left\{\begin{array}{c}\frac{\text { medium memory capacity }}{\left\{h_{1}, h_{2}, h_{4}, h_{6}, h_{7}\right\}}, \frac{\text { high degree of freedom }}{\left\{h_{2}, h_{3}, h_{5}, h_{7}\right\}}, \\ \frac{\text { high repeatability }}{\left\{h_{2}, h_{4}, h_{5}, h_{6}\right\}}\end{array}\right\}$

$\left(F^{*}\right)^{2}($ high cost $)=\left\{\frac{\text { high speed }}{\left\{h_{2}, h_{4}, h_{6}, h_{7}\right\}}, \frac{\text { high load capacity }}{\left\{h_{3}, h_{4}, h_{5}, h_{6}\right\}}, \frac{\text { high repeatability }}{\left\{h_{2}, h_{3}, h_{4}, h_{5}, h_{6}\right\}}\right\}$ 
and

$$
\begin{aligned}
& \left.\left(G^{*}\right)^{2} \text { (medium size }\right)=\left\{\frac{\text { high load capacity }}{\left\{h_{1}, h_{2}, h_{5}, h_{7}\right\}}, \frac{\text { high degree of freedom }}{\left\{h_{1}, h_{3}, h_{4}, h_{6}\right\}}\right\} \\
& \left(G^{*}\right)^{2}(\text { small weight })=\left\{\begin{array}{c}
\frac{\text { medium memory capacity }}{\left\{h_{1}, h_{2}, h_{4}, h_{6}\right\}}, \frac{\text { high speed }}{\left\{h_{1}, h_{4}, h_{6}, h_{7}\right\}}, \\
\frac{\text { high degree of freedom }}{\left\{h_{1}, h_{3}, h_{5}, h_{7}\right\}}
\end{array}\right\} \\
& \left(G^{*}\right)^{2}(\text { high cost })=\left\{\begin{array}{c}
\left.\frac{\text { high repeatability }}{\left\{h_{1}, h_{4}, h_{5}, h_{6}\right\}}, \frac{\text { high load capacity }}{\left\{h_{1}, h_{3}, h_{5}, h_{7}\right\}}\right\}
\end{array}\right.
\end{aligned}
$$

Now $\left[F^{*}, A\right]^{2} \sqcap_{\varepsilon}\left[G^{*}, B\right]^{2}=\left[K^{*}, C\right]$ where $C=A \cup B$. For $c \in C,\left[K^{*}, C\right]$ is as following, $K^{*}($ aluminum material $)=\left\{\frac{\text { high speed }}{\left\{h_{1}, h_{3}, h_{6}, h_{7}\right\}}, \frac{\text { high degree of freedom }}{\left\{h_{1}, h_{2}, h_{3}, h_{4}, h_{5}\right\}}\right\}$ $K^{*}($ medium size $)=\left\{\frac{\text { high load capacity }}{\left\{h_{1}, h_{2}, h_{5}, h_{7}\right\}}, \frac{\text { high degree of freedom }}{\left\{h_{1}, h_{3}, h_{4}, h_{6}\right\}}\right\}$ $K^{*}($ small weight $)=\left\{\begin{array}{c}\frac{\text { medium memory capacity }}{\left\{h_{1}, h_{2}, h_{4}, h_{6}\right\}}, \frac{\text { high speed }}{\left\{h_{1}, h_{4}, h_{6}, h_{7}\right\}}, \\ \frac{\text { high degree of freedom }}{\left\{h_{3}, h_{5}, h_{7}\right\}}, \frac{\text { high repeatability }}{\left\{h_{2}, h_{4}, h_{5}, h_{6}\right\}}\end{array}\right\}$ $K^{*}($ high cost $)=\left\{\frac{\text { high repeatability }}{\left\{h_{4}, h_{5}, h_{6}\right.}, \frac{\text { high speed }}{\left\{h_{2}, h_{4}, h_{6}, h_{7}\right\}}, \frac{\text { high load capacity }}{\left\{h_{3}, h_{5}\right\}}\right\}$

Since $\left(I^{*}\right)^{2}$ and $K^{*}$ are indeed the same results, we conclude the validity Theorem 4.2. Hence $\left(I^{*}\right)^{2}$ or $K^{*}$ are non-appropriate robots for this company in term of the complete sets of primary attributes and corresponding underlying parameters.

(ii) Suppose company looks at the complete set of primary parameters and restricted set of underlying parameters corresponding to common primary parameters of decision makers. The following two methods to obtain the results

$\left(p_{2}\right)$ In the first method company combining both observations by using extendedrestricted union and then takes the complement of it. Then $\left[F^{*}, A\right] \sqcup_{\varepsilon-r}\left[G^{*}, B\right]=\left[I^{*}, C\right]$, where $C=A \cup B$. For all $\alpha \in C,\left[I^{*}, C\right]$ is as following,

$$
\begin{aligned}
& I^{*}(\text { aluminum material })=\left\{\frac{\text { high speed }}{\left\{h_{2}, h_{4}, h_{5}\right\}}, \frac{\text { high degree of freedom }}{\left\{h_{6}, h_{7}\right\}}\right\} \\
& I^{*}(\text { medium size })=\left\{\frac{\text { high load capacity }}{\left\{h_{3}, h_{4}, h_{6}\right\}}, \frac{\text { high degree of freedom }}{\left\{h_{2}, h_{5}, h_{7}\right\}}\right\} \\
& I^{*}(\text { small weight })=\left\{\frac{\text { medium memory capacity }}{\left\{h_{3}, h_{5}, h_{7}\right\}}, \frac{\text { high degree of freedom }}{\left\{h_{1}, h_{2}, h_{4}, h_{6}\right\}}\right\} \\
& I^{*}(\text { high cost })=\left\{\frac{\text { high repeatability }}{\left\{h_{1}, h_{2}, h_{3}, h_{7}\right\}}, \frac{\text { high load capacity }}{\left\{h_{1}, h_{2}, h_{4}, h_{6}, h_{7}\right\}}\right\} . \\
& \text { Then } \\
& \left(I^{*}\right)^{2}(\text { aluminum material })=\left\{\frac{\text { high speed }}{\left\{h_{1}, h_{3}, h_{6}, h_{7}\right\}}, \frac{\text { high degree of freedom }}{\left\{h_{1}, h_{2}, h_{3}, h_{4}, h_{5}\right\}}\right\} \\
& \left(I^{*}\right)^{2}(\text { medium size })=\left\{\frac{\text { high load capacity }}{\left\{h_{1}, h_{2}, h_{5}, h_{7}\right\}}, \frac{\text { high degree of freedom }}{\left\{h_{1}, h_{3}, h_{4}, h_{6}\right\}}\right\} \\
& \left(I^{*}\right)^{2}(\text { small weight })=\left\{\frac{\text { medium memory capacity }}{\left\{h_{1}, h_{2}, h_{4}, h_{6}\right\}}, \frac{\text { high degree of freedom }}{\left\{h_{3}, h_{5}, h_{7}\right\}}\right\} \\
& \left(I^{*}\right)^{2}(\text { high cost })=\left\{\frac{\text { high repeatability }}{\left\{h_{4}, h_{5}, h_{6}\right\}}, \frac{\text { high load capacity }}{\left\{h_{3}, h_{5}\right\}}\right\}
\end{aligned}
$$


These are the most unsuitable robots on the complete set of primary parameters and on restricted sets of underlying parameters corresponding to common primary parameters of decision makers.

$\left(q_{2}\right)$ In this method company first takes complement of both observation $F^{*}$ and $G^{*}$. Then by using extended-restricted intersection company gets the result.

Since $\left[F^{*}, A\right]^{2}$ and $\left[G^{*}, B\right]^{2}$ are calculated in Method $\left(q_{1}\right)$ in $(i)$. Now $\left[F^{*}, A\right]^{2} \sqcap_{\varepsilon-r}$ $\left[G^{*}, B\right]^{2}=\left[K^{*}, C\right]$ where $C=A \cup B$. For $c \in C,\left[K^{*}, C\right]$ is as following,

$K^{*}($ aluminum material $)=\left\{\frac{\text { high speed }}{\left\{h_{1}, h_{3}, h_{6}, h_{7}\right\}}, \frac{\text { high degree of freedom }}{\left\{h_{1}, h_{2}, h_{3}, h_{4}, h_{5}\right\}}\right\}$

$K^{*}($ medium size $)=\left\{\frac{\text { high load capacity }}{\left\{h_{1}, h_{2}, h_{5}, h_{7}\right\}}, \frac{\text { high degree of freedom }}{\left\{h_{1}, h_{3}, h_{4}, h_{6}\right\}}\right\}$

$K^{*}($ small weight $)=\left\{\frac{\text { medium memory capacity }}{\left\{h_{1}, h_{2}, h_{4}, h_{6}\right\}}, \frac{\text { high degree of freedom }}{\left\{h_{3}, h_{5}, h_{7}\right\}}\right\}$

$K^{*}($ high cost $)=\left\{\frac{\text { high speed }}{\left\{h_{2}, h_{4}, h_{6}, h_{7}\right\}}, \frac{\text { high load capacity }}{\left\{h_{3}, h_{4}, h_{5}, h_{6}\right\}}\right\}$

These are the most unsuitable robots on complete set primary parameters and on the restricted sets of underlying parameters corresponding to common primary parameters of decision makers.

Since $\left(I^{*}\right)^{2}$ and $K^{*}$ are indeed the same results, we conclude the validity of $(i)$ in Theorem 4.4. Hence $\left(I^{*}\right)^{2}$ or $K^{*}$ are non-appropriate robots for this company with respect to all primary attributes and restricted underlying parameters corresponding to common primary attributes.

$\left(\Gamma_{2}\right)$ Now the company is interesting to select robots only on common primary attributes of decision makers: small weight, high cost.

(iii) Suppose company looks at both common primary parameters and on restricted underlying parameters corresponding to common primary parameters of decision makers. The following two methods to obtain the results

$\left(p_{3}\right)$ In first method company combining both observations by using restricted union and then takes the complement of it. Then $\left[F^{*}, A\right] \sqcup_{r}\left[G^{*}, B\right]=\left[I^{*}, C\right]$, where $C=A \cap B$. For all $\alpha \in C,\left[I^{*}, C\right]$ is as the following,

$I^{*}($ small weight $)=\left\{\frac{\text { medium memory capacity }}{\left\{h_{3}, h_{5}, h_{7}\right\}}, \frac{\text { high degree of freedom }}{\left\{h_{1}, h_{2}, h_{4}, h_{6}\right\}}\right\}$

$I^{*}($ high cost $)=\left\{\frac{\text { high repeatability }}{\left\{h_{1}, h_{2}, h_{3}, h_{7}\right\}}, \frac{\text { high load capacity }}{\left\{h_{1}, h_{2}, h_{4}, h_{6}, h_{7}\right\}}\right\}$.

Then

$\left(I^{*}\right)^{2}($ small weight $)=\left\{\frac{\text { medium memory capacity }}{\left\{h_{1}, h_{2}, h_{4}, h_{6}\right\}}, \frac{\text { high degree of freedom }}{\left\{h_{3}, h_{5}, h_{7}\right\}}\right\}$

$\left(I^{*}\right)^{2}($ high cost $)=\left\{\frac{\text { high repeatability }}{\left\{h_{4}, h_{5}, h_{6}\right\}}, \frac{\text { high load capacity }}{\left\{h_{3}, h_{5}\right\}}\right\}$

These are the most unsuitable robots on common primary attributes and restricted underlying parameters corresponding to common primary parameters of decision makers.

$\left(q_{3}\right)$ In this method company first takes complement of both observation $F^{*}$ and $G^{*}$. Then by using restricted intersection company gets the result.

Since $\left[F^{*}, A\right]^{2}$ and $\left[G^{*}, B\right]^{2}$ are calculated in Method $\left(q_{1}\right)$ in $(i)$. Now $\left[F^{*}, A\right]^{2} \sqcap_{r}$ $\left[G^{*}, B\right]^{2}=\left[K^{*}, C\right]$ where $C=A \cap B$. For $c \in C,\left[K^{*}, C\right]$ is as following, 


$$
\begin{aligned}
& K^{*}(\text { small weight })=\left\{\begin{array}{c}
\frac{\text { medium memory capacity }}{\left\{h_{1}, h_{2}, h_{4}, h_{6}\right\}}, \\
\frac{\text { high degree of freedom }}{\left\{h_{3}, h_{5}, h_{7}\right\}}
\end{array}\right\} \\
& K^{*}(\text { high cost })=\left\{\frac{\text { high repeatability }}{\left\{h_{4}, h_{5}, h_{6}\right\}}, \frac{\text { high load capacity }}{\left\{h_{3}, h_{5}\right\}}\right\}
\end{aligned}
$$

Since $\left(I^{*}\right)^{2}$ and $K^{*}$ are indeed the same results, we conclude the validity Theorem 4.1 . Hence $\left(I^{*}\right)^{2}$ or $K^{*}$ are non-appropriate robots for this company in terms common primary parameters and common underlying parameters corresponding to common primary parameters of decision makers.

(iv) Suppose company interesting on common primary parameters and on the complete sets of underlying parameters corresponding to common primary parameters of decision makers. The following two methods to obtain the results

$\left(p_{4}\right)$ In the first method company combining both observation by using restrictedextended union and then takes the complement. Then $\left[F^{*}, A\right] \sqcup_{r-\varepsilon}\left[G^{*}, B\right]=\left[I^{*}, C\right]$, where $C=A \cap B$. For all $\alpha \in C,\left[I^{*}, C\right]$ is as following,

$$
\begin{aligned}
& I^{*}(\text { small weight })=\left\{\begin{array}{c}
\frac{\text { medium memory capacity }}{\left\{h_{3}, h_{5}, h_{7}\right\}}, \frac{\text { high speed }}{\left\{h_{2}, h_{3}, h_{5}\right\}}, \\
\frac{\text { high degree of freedom }}{\left\{h_{1}, h_{2}, h_{4}, h_{6}\right\}}, \frac{\text { high repeatability }}{\left\{h_{1}, h_{3}, h_{7}\right\}}
\end{array}\right\} \\
& I^{*}(\text { high cost })=\left\{\begin{array}{c}
\frac{\text { high repeatability }}{\left\{h_{1}, h_{2}, h_{3}, h_{7}\right\}}, \frac{\text { high speed }}{\left\{h_{1}, h_{3}, h_{5}\right\}}, \\
\frac{\text { high load capacity }}{\left\{h_{1}, h_{2}, h_{4}, h_{6}, h_{7}\right\}}
\end{array}\right\} .
\end{aligned}
$$

Then

$$
\begin{aligned}
& \left(I^{*}\right)^{2}(\text { small weight })=\left\{\begin{array}{c}
\frac{\text { medium memory capacity }}{\left\{h_{1}, h_{2}, h_{4}, h_{6}\right\}}, \frac{\text { high speed }}{\left\{h_{1}, h_{4}, h_{6}, h_{7}\right\}}, \\
\frac{\text { high degree of freedom }}{\left\{h_{3}, h_{5}, h_{7}\right\}}, \frac{\text { high repeatability }}{\left\{h_{2}, h_{4}, h_{5}, h_{6}\right\}}
\end{array}\right\} \\
& \left.\left(I^{*}\right)^{2} \text { high cost }\right)=\left\{\begin{array}{c}
\frac{\text { high repeatability }}{\left\{h_{4}, h_{5}, h_{6}\right\}}, \frac{\text { high speed }}{\left\{h_{2}, h_{4}, h_{6}, h_{7}\right\}}, \\
\frac{\text { high load capacity }}{\left\{h_{3}, h_{5}\right\}}
\end{array}\right\}
\end{aligned}
$$

These are the most unsuitable robots on common primary parameters and on the complete sets of underlying parameters corresponding to common primary parameters of decision makers.

$\left(q_{4}\right)$ In this method company first takes the complements of both observation $F^{*}$ and $G^{*}$. Then by using restricted-extended intersection company gets the result.

Since $\left[F^{*}, A\right]^{2}$ and $\left[G^{*}, B\right]^{2}$ are calculated in Method $\left(q_{1}\right)$ in $(i)$. Now $\left[F^{*}, A\right]^{2} \sqcap_{r-\varepsilon}$ $\left[G^{*}, B\right]^{2}=\left[K^{*}, C\right]$ where $C=A \cap B$. For $c \in C,\left[K^{*}, C\right]$ is as following,

$$
\begin{gathered}
K^{*}(\text { small weight })=\left\{\begin{array}{c}
\frac{\text { medium memory capacity }}{\left\{h_{1}, h_{2}, h_{4}, h_{6}\right\}}, \frac{\text { high speed }}{\left\{h_{1}, h_{4}, h_{6}, h_{7}\right\}}, \\
\frac{\text { high degree of freedom }}{\left\{h_{3}, h_{5}, h_{7}\right\}}, \frac{\text { high repeatability }}{\left\{h_{2}, h_{4}, h_{5}, h_{6}\right\}}
\end{array}\right\} \\
K^{*}(\text { high cost })=\left\{\begin{array}{c}
\frac{\text { high repeatability }}{\left\{h_{4}, h_{5}, h_{6}\right\}}, \frac{\text { high speed }}{\left\{h_{2}, h_{4}, h_{6}, h_{7}\right\}}, \\
\frac{\text { high load capacity }}{\left\{h_{3}, h_{5}\right\}}
\end{array}\right\}
\end{gathered}
$$


These are the most unsuitable robots on common primary parameters and on the complete sets of underlying parameters corresponding to common primary parameters of decision makers.

Since $\left(I^{*}\right)^{2}$ and $K^{*}$ are indeed the same results, we conclude the validity of $(i)$ in Theorem 4.3. Hence $\left(I^{*}\right)^{2}$ or $K^{*}$ are non-appropriate robots for this company with respect to common primary parameters and the complete sets of underlying parameters corresponding to common primary parameters of decision makers.

\section{Conclusion}

In this paper, we have presented a study of operations on type- 2 soft sets. We have investigated two different type of empty type- 2 soft sets such as primary empty type-2 soft set and underlying empty type- 2 soft set. We have introduced four new operations (the extension, the restriction, the extension-restriction, the restriction-extension) each on union, intersection and difference. Moreover, we have proved that four type of De Morgan's laws hold in type- 2 soft set theory with respect to these new definitions of union and intersection. These operations can find vast applications in fields like approximate reasoning, decision making, medical diagnosis, market analysis, analysis of funds, dietetics, etc. The advantages of our new operations are noteworthy. As to future research, one can discuss whether these ideas can help to consider type-2 soft sets based decision making problems.

\section{References}

[1] Aktaş, H., Çagman, N. Soft sets and soft groups, Inform. Sci. 177, 2726-2735, 2007.

[2] Ali, M.I., Feng, F., Liu, X., Min, W.K., Shabir, M. On some new operations in soft set theory, Computers and Mathematics with Applications, 57, 1547-1553, 2009.

[3] Ali, M.I., Shabir, M., Naz, M. Algebraic structures of soft sets associated with new operations, Computers and Mathematics with Applications, 61, 2647-2654, 2011.

[4] Ali, M.I., Shabir, M., Samina, Application of L-fuzzy soft sets to semirings, Journal of Intelligent \& Fuzzy Systems, 27, 1731-1742, 2014.

[5] Ali, M.I., Shabir, M. Comments on De Morgan's law in fuzzy soft sets, International Journal of Fuzzy Mathematics, 18, 679-686, 2010.

[6] Ali, M.I., Shabir, M., Shum, K.P. On Soft Ideals over Semigroups, Southeast Asian Bulletin of Mathematics, 34, 595-610, 2010.

[7] Alcantud, J.C.R. A novel algorithm for fuzzy soft set based decision making from multiobserver input parameter data set, Information Fusion, 29, 142-148, 2016.

[8] Atanassov, K. Intuitionistic fuzzy sets, Fuzzy Sets and Systems, 20, 87-96, 1986.

[9] Cagman, N., Enginoglu, S. Soft set theory and uni-int decision making, European Journal of Operational Research, 207, 848-855, 2010.

[10] Chatterjeea, R., Majumdar, P., Samanta, S.K.Type-2 soft sets, Journal of Intelligent \& Fuzzy Systems, 29, 885-898, 2015.

[11] Chen, D., Tsang, E.C.C., Yeung, D.S. Wang, X. The parameterization reduction of soft sets and its applications, Computers \& Mathematics with Applications, 49, 757-763, 2005.

[12] Feng, F., Jun, Y.B., Zhao, X. Soft semirings, Comput. Math. Appl. 56, 2621-2628, 2008.

[13] Hayat, H., Irfan, M.I., Cao, B.Y., Yang, X.P. A new type-2 soft set: Type-2 soft graphs, Advances in Fuzzy Systems, Submitted, 2017.

[14] Jun, Y.B., Lee, K.J., Zhan, J. Soft p-ideals of soft BCI-algebras, Comput. Math. Appl. 58, 2060-2068, 2009.

[15] Maji, P.K., Biswas, R., Roy, A.R. Soft set theory, Computers and Mathematics with Applications, 45, 555-562, 2003.

[16] Maji, P.K., Biswas, R., Roy, A.R. Fuzzy soft sets, J. Fuzzy Math. 9, 589-602, 2001.

[17] Maji, P.K, Roy, A.R. An application of soft sets in a decision making problem, Computers and Mathematics with Applications, 44, 1077-1083, 2002. 
[18] Majumdar, P., Samanta, S.K. Decision making based on similarity measure of vague soft sets, Journal of Intelligent \& Fuzzy Systems, 24, 637-646, 2013.

[19] Molodtsov, D. Soft set theory-first results, Computers and Mathematics with Applications, 37, 19-31, 1999.

[20] Pawlak, Z. Rough sets, Int. J. Comput. Inform. Sci. 11, 341-356, 1982.

[21] Pei, D., Miao, D. From soft sets to information systems, in: X. Hu, Q. Liu, A. Skowron, T.Y. Lin, R.R. Yager, B. Zhang (Eds.), Proceedings of Granular Computing, IEEE, 2, 617-621, 2005.

[22] Sezgin, A., Atagün, A.O. On operations of soft sets, Computers and Mathematics with Applications, 61, 1457-1467, 2011.

[23] Shabir, M., Naz, M. On soft topological spaces, Computers and Mathematics with Applications, 61, 1786-1799, 2011.

[24] Torra, V. Hesitant fuzzy sets, International Journal of Intelligent Systems, 25, 529-539, 2010.

[25] Yu, B., Li, Q. Rough soft set theory applied to lattices and its applications, Journal of Intelligent \& Fuzzy Systems, 6, 3867-3878, 2017.

[26] Zadeh, L.A. Fuzzy Sets, Information and Control, 8, 338-353, 1965. 